\begin{tabular}{|r|l|}
\hline \multicolumn{2}{|c|}{ Statistica Sinica Preprint No: SS-2020-0401 } \\
\hline Title & $\begin{array}{l}\text { Penalized Regression for Multiple Types of Many } \\
\text { Features With Missing Data }\end{array}$ \\
\hline Manuscript ID & SS-2020-0401 \\
\hline URL & http://www.stat.sinica.edu.tw/statistica/ \\
\hline DOI & $10.5705 /$ ss.202020.0401 \\
\hline Complete List of Authors & $\begin{array}{l}\text { Kin Yau Wong, } \\
\text { Donglin Zeng and } \\
\text { Danyu Lin }\end{array}$ \\
\hline Corresponding Author & Kin Yau Wong \\
\hline E-mail & kin-yau.wong@polyu.edu.hk \\
\hline Notice: Accepted version subject to English editing. \\
\hline
\end{tabular}




\title{
PENALIZED REGRESSION FOR MULTIPLE TYPES OF MANY FEATURES WITH MISSING DATA
}

\author{
Kin Yau Wong ${ }^{1}$, Donglin Zeng ${ }^{2}$, and D. Y. Lin $^{2}$ \\ ${ }^{1}$ The Hong Kong Polytechnic University and ${ }^{2}$ The University of North Carolina at Chapel Hill
}

\begin{abstract}
Recent technological advances have made it possible to measure multiple types of many features in biomedical studies. However, some data types or features may not be measured for all study subjects because of cost or other constraints. We use a latent variable model to characterize the relationships across and within data types and to infer missing values from observed data. We develop a penalized-likelihood approach for variable selection and parameter estimation and devise an efficient expectation-maximization (EM) algorithm to implement our approach. We establish the asymptotic properties of the proposed estimators when the number of features increases at a polynomial rate of the sample size. Finally, we demonstrate the usefulness of the proposed methods through extensive simulation studies and provide an application to a motivating multi-platform genomics study.
\end{abstract}

Key words and phrases: Adaptive lasso, Factor models, Integrative analysis, Multi-modality data, Multi-platform genomics studies, Penalized regression

Corresponding author: Kin Yau Wong, Department of Applied Mathematics, The Hong Kong Polytechnic University, Hung Hom, Kowloon, Hong Kong. E-mail: kin-yau.wong@polyu.edu.hk. 


\section{Introduction}

Modern biomedical studies often collect multiple types of data or multi-modality data on a large number of subjects. It is desirable to integrate such data because different modalities play unique roles in complex biological systems. For example, in the study of Alzheimer's disease, the integration of data on magnetic resonance imaging, positron emission tomography, and cerebrospinal fluid can yield more accurate disease classification (Zhang, Shen and The Alzheimer's Disease Neuroimaging Initiative, 2012). In cancer research, different types of genomics data, such as DNA alterations, RNA expressions, and protein expressions, can be integrated to identify disease subtypes and predict patient survival (Shen, Olshen and Ladanyi, 2009; Wang et al., 2012; Hoadley et al., 2014; Wong et al. 2019).

Due to cost or other constraints, certain features are not measured on all study sub-

jects. For example, in The Cancer Genome Atlas (TCGA) (https://cancergenome.nih.gov/), data on multiple types of genomic features, including DNA alterations, methylation profiles, and the expressions of RNA and protein, were collected for over 10,000 patients with 33 cancer types. For a substantial number of the patients, however, data on protein expressions were not generated. As another example, in the Trans-Omics for Precision Medicine (TOPMed) program (https://www.nhlbi.nih.gov/research/resources/nhlbiprecision-medicine-initiative/topmed), whole-genome sequencing data will be available for hundreds of thousands of subjects, but other types of genomics data, such as RNA expressions, methylation profiles, and metabolites, will be available for only a few thousand subjects through ancillary studies of specific diseases. 
It is highly desirable to identify a small subset of features that are associated with the outcome of interest and to estimate the effects of these features. To perform variable selection and estimation with missing data, one may first produce a complete data set and then apply conventional penalized regression methods (Tibshirani, 1996 ; Fan and Li, 2001; Zou, 2006) to the complete data set. Complete data may be obtained through deletion of entries with missing data, mean imputation, and nearest-neighbor imputation (Troyanskaya et al., 2001). Recently, Cai, Cai and Zhang (2016) proposed an imputation method specific to multi-modality data by assuming that the (complete) feature matrix is approximately low-rank; the method is only applicable to a blockwise missing-data pattern, where a data type is either entirely missing or entirely observed on a subject. In general, the two-step approach to variable selection with missing data is inefficient due to discarding available data and ignoring the associations among the observed and missing variables and yields inconsistent estimators when data are not missing completely at random. To accommodate the missing-at-random mechanism, Ibrahim, Zhu and Tang (2008), Garcia, Ibrahim and Zhu (2010), and Jiang, Nguyen and Rao (2015) proposed to model the variables with missing values and perform variable selection using informationcriterion or penalization methods. However, those approaches are intractable when there are many variables with missing values, as in our case.

Regression analysis on large, multi-modality data sets with missing values is highly challenging for two reasons. First, because different types of features tend to be correlated, efficient methods ought to leverage their relationships, but it is difficult to formulate or estimate the intricate relationships among different types of many features. Second, in the presence of missing data, a tractable objective function for estimation is often 
unavailable; for instance, the likelihood function would generally involve integration over many variables and not have a closed form.

To address the aforementioned challenges, we propose a penalized-likelihood approach in which the likelihood involves both an outcome model and a latent factor model for the potentially missing features. The factor model uses a small set of latent factors to explain the associations among features across or within individual data types, effectively reducing the dimensionality of the data. In multi-platform genomics studies, the latent factors can be interpreted as unobserved biological processes that govern the activities of different genomic features; this kind of model has been successfully used to combine multiple types of genomics data in order to understand the interactions among different types of features, recover personal genomics characteristics of cancer patients, and discover cancer subtypes (Shen, Olshen and Ladanyi, 2009; Shen, Wang and Mo, 2013; Lock et al., 2013).

Because the observed-data likelihood involves integration over the features with missing values, direct maximization of the (penalized) likelihood is computationally intensive or even infeasible when the number of features is moderately large. To efficiently compute the penalized estimators, we develop an EM algorithm (Dempster, Laird and Rubin, 1977) which, by making use of the low-dimensional structure of the latent factor model, involves only low-dimensional integration. The algorithm is applicable to general missingdata patterns with a large number of features.

Because the likelihood involves the latent factor model, the total number of nonzero parameters is larger than the number of features. As a result, estimation consistency cannot be established under conventional high-dimensional settings, where the number of features is larger than the sample size. In fact, it is highly challenging to establish the 


\section{MODEL, LIKELIHOOD, AND PENALIZED ESTIMATION}

estimation and selection consistency of our penalized estimators even when the number of features is smaller than but diverges with the sample size. In existing work on large latent factor models, estimation is based on principal components analysis (Bai, 2003; Fan, Liao and Mincheva, 2013; Fan, Liu and Wang, 2018) or maximum likelihood (Bai and Li, 2012; Bai and Liao, 2016). In those cases, the theoretical developments rely heavily on the specific closed-form expressions of the estimators or the likelihood. In our setting, variables may be missing, and the latent factor model is only a part of the full likelihood that generally does not have a closed-form expression. In addition, proofs for estimation and selection consistency of penalized regression methods for complete data (Fan and Peng, 2004; Fan and Lv, 2011) are not applicable, because an essential assumption about the lower bound of the eigenvalues of the information matrix does not hold for the latent factor model.

The rest of this article is structured as follows. In Section 2, we formulate the model and define the maximum penalized-likelihood estimator. In Section 3, we describe the numerical implementation of the proposed methods. In Section 4, we present asymptotic properties of the penalized estimators. In Section 5, we report the results from simulation studies. In Section 6, we provide an application to a TCGA multi-platform genomics data set. We make concluding remarks in Section 7 and relegate theoretical details to the Appendix.

\section{Model, likelihood, and penalized estimation}

Let $Y$ be an outcome variable, $\boldsymbol{X}$ be a vector of covariates, and $\left(\boldsymbol{S}^{(1)}, \ldots, \boldsymbol{S}^{(K)}\right)$ be $K$ types of potentially missing covariates for some $K \geq 1$. Let $\boldsymbol{S}=\left(\boldsymbol{S}^{(1) \mathrm{T}}, \ldots, \boldsymbol{S}^{(K) \mathrm{T}}\right)^{\mathrm{T}}$. 
Suppose that the dimension of $\boldsymbol{X}$ is fixed, whereas the dimension of $\boldsymbol{S}$, denoted by $p_{n}$, may change with the sample size $n$. We specify the following models for $Y$ and $\boldsymbol{S}$ :

$$
\begin{aligned}
Y \mid(\boldsymbol{X}, \boldsymbol{S}) & \sim f\left(\cdot ; \boldsymbol{\alpha}^{\mathrm{T}} \boldsymbol{X}+\boldsymbol{\beta}^{\mathrm{T}} \boldsymbol{S}, \boldsymbol{\xi}\right), \\
\boldsymbol{S}^{(k)} & =\boldsymbol{\Gamma}^{(k)} \boldsymbol{X}+\boldsymbol{\Psi}^{(0, k)} \boldsymbol{U}^{(0)}+\boldsymbol{\Psi}^{(k)} \boldsymbol{U}^{(k)}+\boldsymbol{\epsilon}^{(k)} \quad \text { for } k=1, \ldots, K,
\end{aligned}
$$

where $f$ is a parametric density function, $\boldsymbol{\alpha}$ and $\boldsymbol{\beta}$ are vectors of regression parameters, $\boldsymbol{\xi}$ is a vector of low-dimensional nuisance parameters, $\boldsymbol{U}^{(k)}$ is a multivariate standard-normal latent variables with dimension $r_{k}\left(r_{k} \geq 0\right)$ for $k=0, \ldots, K,\left(\boldsymbol{\Gamma}^{(k)}, \Psi^{(0, k)}, \Psi^{(k)}\right)_{k=1, \ldots, K}$ are matrices of regression parameters, and $\left(\boldsymbol{\epsilon}^{(1)}, \ldots, \boldsymbol{\epsilon}^{(K)}\right)$ are zero-mean normal variables with independent components. The variables $\left(\boldsymbol{U}^{(0)}, \ldots, \boldsymbol{U}^{(K)}, \boldsymbol{\epsilon}^{(1)}, \ldots, \boldsymbol{\epsilon}^{(K)}\right)$ are mutually independent. The numbers of latent variables $\left(r_{0}, \ldots, r_{K}\right)$ are chosen to be much smaller than the dimension of each type of features. To ensure model identifiability, we set $\psi_{j l}^{(0,1)}=0$ and $\psi_{j l}^{(k)}=0$ for $l>j$ and $k=1, \ldots, K$, where $\psi_{j l}^{(0,1)}$ and $\psi_{j l}^{(k)}$ are the $(j, l)$ th elements of $\Psi^{(0,1)}$ and $\Psi^{(k)}$, respectively. Also, we assume that $\psi_{j j}^{(0,1)}>0$ for $j=1, \ldots, r_{0}$ and $\psi_{j j}^{(k)}>0$ for $j=1, \ldots, r_{k}$ and $k=1, \ldots, K$. These conditions are analogous to condition (IC5) of Bai and Li (2012). They are satisfied if the first $r_{k}$ components of the $k$ th feature type depend on all corresponding type-specific latent variables $\boldsymbol{U}^{(k)}$, the first $r_{0}$ components of the first feature type depend on all common latent variables $\boldsymbol{U}^{(0)}$, and the corresponding vectors of factor loadings are linearly independent; in this case, the latent variables can be transformed to yield the desired structure for the factor loading matrices. If these conditions are in doubt, we may refit the model with a different ordering of features. The model of $Y$, hereafter referred to as the outcome model, includes many common models, such as the linear and logistic regression models, as special cases. The 


\section{MODEL, LIKELIHOOD, AND PENALIZED ESTIMATION}

model of $\boldsymbol{S}$ is a latent factor model, which assumes that $\boldsymbol{S}$ (conditional on $\boldsymbol{X}$ ) follows a multivariate normal distribution, and the associations among the multi-modality features are induced by a small set of unobserved latent factors $\boldsymbol{U} \equiv\left(\boldsymbol{U}^{(0) \mathrm{T}}, \ldots, \boldsymbol{U}^{(K) \mathrm{T}}\right)^{\mathrm{T}}$.

The factor model captures the associations among features across different data types as well as within individual data types. The set of latent variables $\boldsymbol{U}^{(0)}$ is shared among all data types and induces associations across all features. For $k=1, \ldots, K$, the set of latent variables $\boldsymbol{U}^{(k)}$ is shared only among components of $\boldsymbol{S}^{(k)}$ and captures the associations among features of this data type that are not explained by $\boldsymbol{U}^{(0)}$. The factor model is plausible for many applications in which features of individual or multiple types share common sources of variability. For example, in multi-platform cancer genomics studies, different types of genomic features are commonly affected by major biological processes, such as growth suppressor evasion and cell death resistance (Hanahan and Weinberg, 2011); associations induced by such processes can be captured by $\boldsymbol{U}^{(0)}$. By contrast, some biological processes, such as miRNA regulation, may alter the expression of genes with no effect on other types of features, such as mutations; associations induced by such processes can be captured by the type-specific latent variables $\boldsymbol{U}^{(k)}(k=1, \ldots, K)$.

We allow each component of $\boldsymbol{S} \equiv\left(S_{1}, \ldots, S_{p_{n}}\right)^{\mathrm{T}}$ to be missing and use $M_{j}$ to indicate, by the values 1 versus 0 , whether $S_{j}$ is observed or missing $\left(j=1, \ldots, p_{n}\right)$. We assume that $\boldsymbol{S}$ is missing at random, such that $\boldsymbol{M} \equiv\left(M_{1}, \ldots, M_{p_{n}}\right)^{\mathrm{T}}$ is independent of $\boldsymbol{S}$ conditional on $(Y, \boldsymbol{X}, \overline{\boldsymbol{S}})$, where $\overline{\boldsymbol{S}}=\left\{S_{j}: P\left(M_{j}=1\right)=1\right\}$. This assumption holds when missing data are introduced by design, where subjects with specific values of $(Y, \boldsymbol{X}, \overline{\boldsymbol{S}})$ are selected for measurements of components of $\boldsymbol{S}$ (not included in $\overline{\boldsymbol{S}}$ ). The assumption also holds when missing data arise from random technical errors in the data-collection process that 
are independent of the data.

For a random sample of size $n$, the observed data consist of $\left(Y_{i}, \boldsymbol{X}_{i}, \boldsymbol{M}_{i}, \boldsymbol{M}_{i} \circ \boldsymbol{S}_{i}\right)$ $(i=1, \ldots, n)$, where $\circ$ denotes componentwise multiplication. Let $r=\sum_{k=0}^{K} r_{k}, \boldsymbol{\Gamma}=$ $\left(\boldsymbol{\Gamma}^{(1) \mathrm{T}}, \ldots, \boldsymbol{\Gamma}^{(K) \mathrm{T}}\right)^{\mathrm{T}}, \boldsymbol{\Psi}$ be a $\left(p_{n} \times r\right)$ matrix with

$$
\boldsymbol{\Psi}=\left(\begin{array}{ccccc}
\boldsymbol{\Psi}^{(0,1)} & \boldsymbol{\Psi}^{(1)} & \mathbf{0} & \ldots & \mathbf{0} \\
\boldsymbol{\Psi}^{(0,2)} & \mathbf{0} & \boldsymbol{\Psi}^{(2)} & \ldots & \mathbf{0} \\
\vdots & \vdots & & \ddots & \vdots \\
\boldsymbol{\Psi}^{(0, K)} & \mathbf{0} & \ldots & \mathbf{0} & \boldsymbol{\Psi}^{(K)}
\end{array}\right)
$$

and $\boldsymbol{\Sigma}$ be a $\left(p_{n} \times p_{n}\right)$ diagonal matrix with the diagonal elements being the variances of the components of $\left(\boldsymbol{\epsilon}^{(1)}, \ldots, \boldsymbol{\epsilon}^{(K)}\right)$. Let $\boldsymbol{\theta} \equiv(\boldsymbol{\alpha}, \boldsymbol{\beta}, \boldsymbol{\xi}, \boldsymbol{\Gamma}, \boldsymbol{\Psi}, \boldsymbol{\Sigma})$ denote the collection of all parameters. The observed-data $\log$-likelihood function concerning $\boldsymbol{\theta}$ is

$$
\ell_{n}(\boldsymbol{\theta})=\sum_{i=1}^{n} \log \int f\left(Y_{i} ; \boldsymbol{\alpha}^{\mathrm{T}} \boldsymbol{X}_{i}+\boldsymbol{\beta}^{\mathrm{T}} \boldsymbol{S}_{i}, \boldsymbol{\xi}\right) \phi\left(\boldsymbol{S}_{i} ; \boldsymbol{\Gamma} \boldsymbol{X}_{i}, \boldsymbol{\Psi} \Psi^{\mathrm{T}}+\boldsymbol{\Sigma}\right) \mathrm{d} \boldsymbol{S}_{i}^{(M)}
$$

where $\boldsymbol{S}_{i}^{(M)}$ is the vector of the missing components of $\boldsymbol{S}_{i}$, and $\phi(\cdot ; \boldsymbol{\mu}, \boldsymbol{\Omega})$ is the density of the multivariate normal distribution with mean $\boldsymbol{\mu}$ and covariance matrix $\boldsymbol{\Omega}$. We propose to estimate $\boldsymbol{\theta}$ using maximum penalized-likelihood estimation with an adaptive-lasso (Zou, 2006 ) penalty on $\boldsymbol{\beta}$. Specifically, the penalized estimator $\widehat{\boldsymbol{\theta}} \equiv(\widehat{\boldsymbol{\alpha}}, \widehat{\boldsymbol{\beta}}, \widehat{\boldsymbol{\xi}}, \widehat{\boldsymbol{\Gamma}}, \widehat{\boldsymbol{\Psi}}, \widehat{\boldsymbol{\Sigma}})$ maximizes

$$
p \ell_{n}(\boldsymbol{\theta}) \equiv \ell_{n}(\boldsymbol{\theta})-n \lambda_{n} \sum_{j=1}^{p_{n}} w_{j}\left|\beta_{j}\right|
$$

where $\lambda_{n}$ is a tuning parameter, and $w_{j} \equiv\left|\widetilde{\beta}_{j}\right|^{-1}$ is a weight term derived from an initial estimator $\widetilde{\beta}_{j}\left(j=1, \ldots, p_{n}\right)$.

In general, the likelihood involves the conditional distribution of $Y$ given the observed components of $\boldsymbol{S}$ (and $\boldsymbol{X}$ ) and the distribution of the observed components of $\boldsymbol{S}$. When 
the data are complete, the conditional distribution of $Y$ involves $(\boldsymbol{\alpha}, \boldsymbol{\beta}, \boldsymbol{\xi})$ only, and the distribution of $\boldsymbol{S}$ involves $(\boldsymbol{\Gamma}, \boldsymbol{\Psi}, \boldsymbol{\Sigma})$ only, such that the two sets of parameters can be estimated separately. With missing data, however, the conditional distribution of $Y$ given the observed components of $\boldsymbol{S}$ involves $(\boldsymbol{\alpha}, \boldsymbol{\beta}, \boldsymbol{\xi})$ and functions of $(\boldsymbol{\Gamma}, \boldsymbol{\Psi}, \boldsymbol{\Sigma})$ that capture the relationship between the missing and observed components of $\boldsymbol{S}$. Therefore, valid estimation and variable selection for the outcome model ought to properly account for the relationships among different components of $\boldsymbol{S}$. Mean imputation completely ignores these relationships, and single imputation based on the observed components of $\boldsymbol{S}$ alone may be biased when the missing-data mechanism depends on $(Y, \boldsymbol{X})$; both approaches may yield inefficient or inconsistent estimation of the outcome model.

To obtain the initial estimators $\widetilde{\beta}_{j}\left(j=1, \ldots, p_{n}\right)$, one may maximize $\ell_{n}(\cdot)$ with an $L_{1}$ or $L_{2}$ penalty on $\boldsymbol{\beta}$. However, this approach involves an extra step of tuning parameter selection and is computationally intensive due to the presence of missing data. An alternative approach is to fit a "marginal" regression model of $Y$ against $\left(\boldsymbol{X}, S_{j}\right)$ for each $j$ and use the regression parameter estimator for $S_{j}$ as the initial estimator $\widetilde{\beta}_{j}$ $\left(j=1, \ldots, p_{n}\right)$. In each model, we assume that $S_{j}$ follows a linear regression model with covariates $\boldsymbol{X}$. Involving only a single incomplete independent variable, the model can be easily estimated using the EM algorithm. We adopt the marginal approach because it is computationally efficient and does not require tuning. We expect the marginal approach to perform well when the marginal effects of $\boldsymbol{S}$ have a sparsity structure similar to that of the conditional effects. 


\section{Computation of the penalized estimators}

It is convenient to introduce the notation: $\mathcal{M}_{i}=\left\{j: M_{i j}=0\right\}$, and $\mathcal{M}_{i}^{\mathrm{C}}=\left\{1, \ldots, p_{n}\right\} \backslash \mathcal{M}_{i}$. Here and in the sequel, $b_{i j}$ denotes the $j$ th component of the vector $\boldsymbol{b}_{i}$. Let $\boldsymbol{S}_{i}^{(O)}=\left(\boldsymbol{S}_{i}\right)_{\mathcal{M}_{i}^{\mathrm{C}}}$, $\boldsymbol{\beta}_{i}^{(M)}=\boldsymbol{\beta}_{\mathcal{M}_{i}}, \boldsymbol{\beta}_{i}^{(O)}=\boldsymbol{\beta}_{\mathcal{M}_{i}^{\mathrm{C}}}, \boldsymbol{\epsilon}_{i}^{(M)}=\left(\boldsymbol{\epsilon}_{i}\right)_{\mathcal{M}_{i}}, \boldsymbol{\Gamma}_{i}^{(M)}=\boldsymbol{\Gamma}_{\mathcal{M}_{i}}$, and $\boldsymbol{\Psi}_{i}^{(M)}=\boldsymbol{\Psi}_{\mathcal{M}_{i}}$, where $\boldsymbol{b}_{\mathcal{A}}$ is the vector that consists of all the components of $\boldsymbol{b}$ indexed by $\mathcal{A}$, and $\boldsymbol{B}_{\mathcal{A}}$ is the matrix that consists of all the rows of $\boldsymbol{B}$ indexed by $\mathcal{A}$. By the definition of the factor model, the likelihood function is proportional to

$$
\prod_{i=1}^{n} \int f\left(Y_{i} ; \boldsymbol{\alpha}^{\mathrm{T}} \boldsymbol{X}_{i}+\boldsymbol{\beta}^{\mathrm{T}} \boldsymbol{S}_{i}, \boldsymbol{\xi}\right) \prod_{j=1}^{p_{n}} \sigma_{j}^{-1} \exp \left\{-\frac{\left(S_{i j}-\boldsymbol{\gamma}_{j}^{\mathrm{T}} \boldsymbol{X}_{i}-\boldsymbol{\psi}_{j}^{\mathrm{T}} \boldsymbol{U}_{i}\right)^{2}}{2 \sigma_{j}^{2}}\right\} e^{-\frac{1}{2} \boldsymbol{U}_{i}^{\mathrm{T}} \boldsymbol{U}_{i}} \mathrm{~d}\left(\boldsymbol{U}_{i}, \boldsymbol{S}_{i}^{(M)}\right),
$$

where $\boldsymbol{\gamma}_{j}^{\mathrm{T}}$ and $\boldsymbol{\psi}_{j}^{\mathrm{T}}$ are the $j$ th rows of $\boldsymbol{\Gamma}$ and $\boldsymbol{\Psi}$, respectively, and $\sigma_{j}^{2}$ is the $j$ th diagonal element of $\boldsymbol{\Sigma}$. With $S_{i j}=\boldsymbol{\gamma}_{j}^{\mathrm{T}} \boldsymbol{X}_{i}+\boldsymbol{\psi}_{j}^{\mathrm{T}} \boldsymbol{U}_{i}+\epsilon_{i j}$ for $M_{i j}=0$, the above expression becomes

$$
\begin{aligned}
& \prod_{i=1}^{n} \int f\left(Y_{i} ; \boldsymbol{\alpha}^{\mathrm{T}} \boldsymbol{X}_{i}+\boldsymbol{\beta}_{i}^{(O) \mathrm{T}} \boldsymbol{S}_{i}^{(O)}+\boldsymbol{\beta}_{i}^{(M) \mathrm{T}} \boldsymbol{\Gamma}_{i}^{(M)} \boldsymbol{X}_{i}+\boldsymbol{\beta}_{i}^{(M) \mathrm{T}} \boldsymbol{\Psi}_{i}^{(M)} \boldsymbol{U}_{i}+\boldsymbol{\beta}_{i}^{(M) \mathrm{T}} \boldsymbol{\epsilon}_{i}^{(M)}, \boldsymbol{\xi}\right) \\
& \times \prod_{j=1}^{p_{n}} \sigma_{j}^{-1} \exp \left[-\frac{1}{2 \sigma_{j}^{2}}\left\{M_{i j}\left(S_{i j}-\boldsymbol{\gamma}_{j}^{\mathrm{T}} \boldsymbol{X}_{i}-\boldsymbol{\psi}_{j}^{\mathrm{T}} \boldsymbol{U}_{i}\right)^{2}+\left(1-M_{i j}\right) \epsilon_{i j}^{2}\right\}\right] e^{-\frac{1}{2} \boldsymbol{U}_{i}^{\mathrm{T}} \boldsymbol{U}_{i}} \mathrm{~d}\left(\boldsymbol{U}_{i}, \boldsymbol{\epsilon}_{i}^{(M)}\right) .
\end{aligned}
$$

To obtain the penalized estimators, we adopt an EM algorithm with $\left(\boldsymbol{U}_{i}, \boldsymbol{\epsilon}_{i}^{(M)}\right)(i=$ $1, \ldots, n)$ as missing data; the algorithm iterates between the E-step and M-step described below until convergence. In contrast to direct maximization of the log-likelihood function, the EM algorithm avoids inversion of large matrices and involves numerical integration of lower dimensions.

In the E-step, we calculate the conditional expectation of functions of $\left(\boldsymbol{U}_{i}, \boldsymbol{\epsilon}_{i}^{(M)}\right)$ that are involved in the M-step. Because all functions of $\boldsymbol{\epsilon}_{i}^{(M)}$ that are involved in the M-step are linear or quadratic, we only need to calculate the conditional expectation of functions 
of $\boldsymbol{U}_{i}$ and a one-dimensional linear transformation of $\boldsymbol{\epsilon}_{i}^{(M)}$. Let $p_{i}^{(M)}$ be the dimension of $\boldsymbol{\epsilon}_{i}^{(M)}, c_{i}=\left\{\sum_{j=1}^{p_{i}^{(M)}}\left(\beta_{i j}^{(M)} \sigma_{i j}^{(M)}\right)^{2}\right\}^{1 / 2}$, and $\widetilde{\epsilon}_{i}=\boldsymbol{\beta}_{i}^{(M) \mathrm{T}} \boldsymbol{\epsilon}_{i}^{(M)}$. Because $\widetilde{\epsilon}_{i}$ is zero-mean normal with variance $c_{i}$ and is independent of $\boldsymbol{U}_{i}$ and $\left\{\epsilon_{i j}: j \in \mathcal{M}_{i}^{\mathrm{C}}\right\}$, the joint density function of $\left(Y_{i}, \boldsymbol{S}_{i}, \boldsymbol{U}_{i}, \widetilde{\epsilon}_{i}\right)$ is proportional to

$$
\begin{aligned}
f\left(Y_{i}, \boldsymbol{S}_{i}, \boldsymbol{U}_{i}, \widetilde{\epsilon}_{i} ; \boldsymbol{X}_{i}\right) \equiv & f\left(Y_{i} ; \boldsymbol{\alpha}^{\mathrm{T}} \boldsymbol{X}_{i}+\boldsymbol{\beta}_{i}^{(O) \mathrm{T}} \boldsymbol{S}_{i}^{(O)}+\boldsymbol{\beta}_{i}^{(M) \mathrm{T}} \boldsymbol{\Gamma}_{i}^{(M)} \boldsymbol{X}_{i}+\boldsymbol{\beta}_{i}^{(M) \mathrm{T}} \boldsymbol{\Psi}_{i}^{(M)} \boldsymbol{U}_{i}+\widetilde{\epsilon}_{i}, \boldsymbol{\xi}\right) \\
& \times \exp \left\{-\sum_{j \in \mathcal{M}_{i}^{\mathrm{C}}} \frac{1}{2 \sigma_{j}^{2}}\left(\boldsymbol{S}_{j}-\boldsymbol{\gamma}_{j}^{\mathrm{T}} \boldsymbol{X}_{i}-\boldsymbol{\psi}_{j}^{\mathrm{T}} \boldsymbol{U}_{i}\right)^{2}-\frac{\widetilde{\epsilon}_{i}^{2}}{2 c_{i}^{2}}-\frac{1}{2} \boldsymbol{U}_{i}^{\mathrm{T}} \boldsymbol{U}_{i}\right\} .
\end{aligned}
$$

The conditional expectation of any function $g$ of $\left(\boldsymbol{U}_{i}, \widetilde{\epsilon}_{i}\right)$ given the observed data is

$$
\mathcal{C}^{-1} \int g\left(\boldsymbol{U}_{i}, \widetilde{\epsilon}_{i}\right) f\left(Y_{i}, \boldsymbol{S}_{i}, \boldsymbol{U}_{i}, \widetilde{\epsilon}_{i} ; \boldsymbol{X}_{i}\right) \mathrm{d}\left(\boldsymbol{U}_{i}, \widetilde{\epsilon}_{i}\right)
$$

where $\mathcal{C}$ equals the above integral evaluated at $g(\cdot, \cdot)=1$. In contrast to the $\left(r+p_{i}^{(M)}\right)$ dimensional integration in $(3.3)$, the integration in $(3.4)$ is of dimension $(r+1)$ only. To approximate (3.4), we extend the approach of Liu and Pierce (1994) to the multivariate setting and write 3.4 as

$$
\int w(\boldsymbol{v}) e^{-\frac{1}{2}\left(\boldsymbol{v}-\boldsymbol{\mu}_{v}\right)^{\mathrm{T}} \boldsymbol{H}_{v}^{-1}\left(\boldsymbol{v}-\boldsymbol{\mu}_{v}\right)} \mathrm{d} \boldsymbol{v}
$$

where $\boldsymbol{v}=\left(\boldsymbol{U}_{i}^{\mathrm{T}}, \widetilde{\epsilon}_{i}\right)^{\mathrm{T}}, w(\boldsymbol{v})=\mathcal{C}^{-1} g\left(\boldsymbol{U}_{i}, \widetilde{\epsilon}_{i}\right) f\left(Y_{i}, \boldsymbol{S}_{i}, \boldsymbol{U}_{i}, \widetilde{\epsilon}_{i} ; \boldsymbol{X}_{i}\right) e^{\frac{1}{2}\left(\boldsymbol{v}-\boldsymbol{\mu}_{v}\right)^{\mathrm{T}} \boldsymbol{H}_{v}^{-1}\left(\boldsymbol{v}-\boldsymbol{\mu}_{v}\right)}, \boldsymbol{\mu}_{v}$ is the maximizer of $f\left(Y_{i}, \boldsymbol{S}_{i}, \boldsymbol{U}_{i}, \widetilde{\epsilon}_{i} ; \boldsymbol{X}_{i}\right)$ with respect to $\left(\boldsymbol{U}_{i}, \widetilde{\epsilon}_{i}\right)$, and $\boldsymbol{H}_{v}$ is the Hessian matrix of $-\log f\left(Y_{i}, \boldsymbol{S}_{i}, \boldsymbol{U}_{i}, \widetilde{\epsilon}_{i} ; \boldsymbol{X}_{i}\right)$ with respect to $\left(\boldsymbol{U}_{i}, \widetilde{\epsilon}_{i}\right)$ evaluated at $\boldsymbol{\mu}_{v}$. Then, we can approximate (3.5) by the sparse-grid multivariate Gauss-Hermite quadrature (Heiss and Winschel, 2008). Unlike a conventional multivariate quadrature, where the number of nodes increases exponentially with the dimension of integration under a fixed level of accuracy, the number of nodes under the sparse-grid quadrature increases only polynomially with the dimension. All functions of $\boldsymbol{\epsilon}_{i}^{(M)}$ that are involved in the M-step can be 
obtained from the first and second moments of $\widetilde{\epsilon}_{i}$. The relationship between the moments of $\left(\boldsymbol{U}_{i}, \boldsymbol{\epsilon}_{i}^{(M)}\right)$ and the moments of $\left(\boldsymbol{U}_{i}, \widetilde{\epsilon}_{i}\right)$ is given in Appendix A.1.

To perform the M-step, we obtain a local quadratic approximation to the log-likelihood of the outcome model. For $i=1, \ldots, n$, let $\widehat{\eta}_{i}=\boldsymbol{\alpha}^{\mathrm{T}} \boldsymbol{X}_{i}+\boldsymbol{\beta}_{i}^{(O) \mathrm{T}} \boldsymbol{S}_{i}^{(O)}+\boldsymbol{\beta}_{i}^{(M) \mathrm{T}} \boldsymbol{\Gamma}_{i}^{(M)} \boldsymbol{X}_{i}+$ $\boldsymbol{\beta}_{i}^{(M) \mathrm{T}} \boldsymbol{\Psi}_{i}^{(M)} \widehat{\mathrm{E}}\left(\boldsymbol{U}_{i}\right)+\widehat{\mathrm{E}}\left(\widetilde{\epsilon}_{i}\right)$, where $\widehat{\mathrm{E}}(\cdot)$ is the conditional expectation obtained from the E-step, and the parameters are evaluated at the estimators obtained from the previous M-step (or the initial estimators for the first iteration). By the Taylor series expansion of $\log f\left(Y_{i} ; \eta, \boldsymbol{\xi}\right)$ at $\eta=\widehat{\eta}_{i}$, we can approximate $\log f\left(Y_{i} ; \eta, \boldsymbol{\xi}\right)$ by $-\left(z_{i}-\eta\right)^{2} /\left(2 s_{i}^{2}\right)$ up to a constant term, where $s_{i}^{2}=-\partial^{2} \log f\left(Y_{i} ; \eta, \boldsymbol{\xi}\right) /\left.\partial \eta^{2}\right|_{\eta=\widehat{\eta}_{i}}$, and $z_{i}=\widehat{\eta}_{i}+s_{i}^{-2} \partial \log f\left(Y_{i} ; \eta, \boldsymbol{\xi}\right) /\left.\partial \eta\right|_{\eta=\widehat{\eta}_{i}}$.

In the M-step, we first update the parameters in the latent factor model in a coordinatewise fashion by maximizing

$$
\begin{aligned}
- & \sum_{i=1}^{n} \widehat{\mathrm{E}}\left\{\frac{M_{i j}}{2 \sigma_{j}^{2}}\left(S_{i j}-\boldsymbol{\gamma}_{j}^{\mathrm{T}} \boldsymbol{X}_{i}-\boldsymbol{\psi}_{j}^{\mathrm{T}} \boldsymbol{U}_{i}\right)^{2}\right. \\
& \left.+\frac{1-M_{i j}}{2 s_{i}^{2}}\left(z_{i}-\boldsymbol{\alpha}^{\mathrm{T}} \boldsymbol{X}_{i}-\boldsymbol{\beta}_{i}^{(O) \mathrm{T}} \boldsymbol{S}_{i}^{(O)}-\boldsymbol{\beta}_{i}^{(M) \mathrm{T}} \boldsymbol{\Gamma}_{i}^{(M)} \boldsymbol{X}_{i}-\boldsymbol{\beta}_{i}^{(M) \mathrm{T}} \boldsymbol{\Psi}_{i}^{(M)} \boldsymbol{U}_{i}-\widetilde{\epsilon}_{i}\right)^{2}\right\}
\end{aligned}
$$

with respect to $\left(\boldsymbol{\gamma}_{j}, \boldsymbol{\psi}_{j}, \sigma_{j}^{2}\right)$ in turn for $j=1, \ldots, p_{n}$, where the remaining parameters are fixed at the current estimators. Note that if $S_{j}$ belongs to the $k$ th data type $(j=$ $\left.1, \ldots, p_{n} ; k=1, \ldots, K\right)$, then only the components of $\boldsymbol{\psi}_{j}$ that correspond to $\boldsymbol{U}^{(0)}$ and $\boldsymbol{U}^{(k)}$ need to be updated; the remaining components are set to 0. Also, under the identifiability conditions, the upper triangular elements of $\Psi^{(0,1)}$ and $\Psi^{(1)}, \ldots, \Psi^{(K)}$ are set to 0 . Then, we update $\boldsymbol{\alpha}$ and $\boldsymbol{\beta}$ by maximizing

$$
\sum_{i=1}^{n} \frac{z_{i}}{s_{i}^{2}}\left\{\boldsymbol{\alpha}^{\mathrm{T}} \boldsymbol{X}_{i}+\boldsymbol{\beta}^{\mathrm{T}} \widehat{\mathrm{E}}\left(\widetilde{\boldsymbol{S}}_{i}\right)\right\}-\frac{1}{2 s_{i}^{2}}\left(\boldsymbol{\alpha}^{\mathrm{T}}, \boldsymbol{\beta}^{\mathrm{T}}\right)\left(\begin{array}{cc}
\boldsymbol{X}_{i} \boldsymbol{X}_{i}^{\mathrm{T}} & \boldsymbol{X}_{i} \widehat{\mathrm{E}}\left(\widetilde{\boldsymbol{S}}_{i}^{\mathrm{T}}\right) \\
\widehat{\mathrm{E}}\left(\widetilde{\boldsymbol{S}}_{i}\right) \boldsymbol{X}_{i}^{\mathrm{T}} & \widehat{\mathrm{E}}\left(\widetilde{\boldsymbol{S}}_{i} \widetilde{\boldsymbol{S}}_{i}^{\mathrm{T}}\right)
\end{array}\right)\left(\begin{array}{c}
\boldsymbol{\alpha} \\
\boldsymbol{\beta}
\end{array}\right)-\lambda_{n}|\boldsymbol{w} \circ \boldsymbol{\beta}|,
$$


where $\widetilde{\boldsymbol{S}}_{i}=\boldsymbol{M}_{i} \circ \boldsymbol{S}_{i}+\left(\mathbf{1}-\boldsymbol{M}_{i}\right) \circ\left(\widehat{\boldsymbol{\Gamma}} \boldsymbol{X}_{i}+\widehat{\boldsymbol{\Psi}} \boldsymbol{U}_{i}+\boldsymbol{\epsilon}_{i}\right)$, and $(\widehat{\boldsymbol{\Gamma}}, \widehat{\boldsymbol{\Psi}})$ are the current estimators of $(\boldsymbol{\Gamma}, \Psi)$. The estimators of $(\boldsymbol{\alpha}, \boldsymbol{\beta})$ can be efficiently computed using the coordinatedescent algorithm (Friedman, Hastie and Tibshirani, 2010) for complete data. Finally, we update the estimator of $\boldsymbol{\xi}$ by maximizing the conditional expected log-likelihood with other parameters evaluated at the current estimators.

To obtain more stable estimators, we adopt the pathwise coordinate-descent approach of Friedman, Hastie and Tibshirani (2010): instead of directly computing the penalized estimators at the selected value of $\lambda_{n}$, we perform the estimation for a sequence of decreasing values of $\lambda_{n}$ up to the selected value. The sequence starts at $\lambda_{\max }$, the smallest value of $\lambda_{n}$ under which all estimators of $\boldsymbol{\beta}$ are zero. In particular, $\lambda_{\max }=\max _{j} w_{j}^{-1}\left|n^{-1} \sum_{i=1}^{n} s_{i}^{-2}\left(z_{i}-\boldsymbol{\alpha}^{\mathrm{T}} \boldsymbol{X}_{i}\right) \widehat{\mathrm{E}}\left(\widetilde{S}_{i j}\right)\right|$, where $\boldsymbol{\alpha}$ and parameters in $s_{i}, z_{i}$, and $\widehat{\mathrm{E}}(\cdot)$ are evaluated at the maximum likelihood estimators (MLE) under $\boldsymbol{\beta}=\mathbf{0}$. For the linear outcome model, the estimator of the error variance may vary greatly over different values of $\lambda_{n}$, and therefore the estimators of $\boldsymbol{\beta}$ that are obtained from maximizing (3.6) may be unstable over different values of $\lambda_{n}$. To overcome this problem, we maximize (3.6) with $s_{i}$ set to 1 in each M-step and also set $s_{i}=1$ in the calculation of $\lambda_{\max }$.

\section{Asymptotic properties of the penalized estimators}

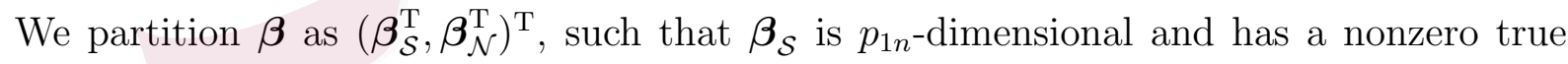
value, and $\boldsymbol{\beta}_{\mathcal{N}}$ has a true value of 0 . Write $\boldsymbol{w}=\left(w_{1}, \ldots, w_{p_{n}}\right)^{\mathrm{T}}$, and partition $\boldsymbol{w}=$ $\left(\boldsymbol{w}_{\mathcal{S}}^{\mathrm{T}}, \boldsymbol{w}_{\mathcal{N}}^{\mathrm{T}}\right)^{\mathrm{T}}$ and $\boldsymbol{S}=\left(\boldsymbol{S}_{\mathcal{S}}^{\mathrm{T}}, \boldsymbol{S}_{\mathcal{N}}^{\mathrm{T}}\right)^{\mathrm{T}}$ to conform with the partitioning of $\boldsymbol{\beta}$. Assume that $p_{n}=O\left(n^{\kappa}\right)$ for some positive $\kappa<1 / 5$ and $p_{1 n}=O\left(p_{n}\right)$.

Let $Z(\eta, \boldsymbol{\xi})=\partial \log f(Y ; \eta, \boldsymbol{\xi}) / \partial \eta, \dot{\ell}_{\alpha}^{(C)}(\boldsymbol{\theta})=Z\left(\boldsymbol{\alpha}^{\mathrm{T}} \boldsymbol{X}+\boldsymbol{\beta}^{\mathrm{T}} \boldsymbol{S}, \boldsymbol{\xi}\right) \boldsymbol{X}, \dot{\boldsymbol{\ell}}_{\beta_{\mathcal{S}}}^{(C)}(\boldsymbol{\theta})=Z\left(\boldsymbol{\alpha}^{\mathrm{T}} \boldsymbol{X}+\right.$ 
$\left.\boldsymbol{\beta}^{\mathrm{T}} \boldsymbol{S}, \boldsymbol{\xi}\right) \boldsymbol{S}_{\mathcal{S}}, \dot{\boldsymbol{\ell}}_{\beta_{\mathcal{N}}}^{(C)}(\boldsymbol{\theta})=Z\left(\boldsymbol{\alpha}^{\mathrm{T}} \boldsymbol{X}+\boldsymbol{\beta}^{\mathrm{T}} \boldsymbol{S}, \boldsymbol{\xi}\right) \boldsymbol{S}_{\mathcal{N}}, \dot{\boldsymbol{\ell}}_{\xi}^{(C)}(\boldsymbol{\theta})=\partial \log f\left(Y ; \boldsymbol{\alpha}^{\mathrm{T}} \boldsymbol{X}+\boldsymbol{\beta}^{\mathrm{T}} \boldsymbol{S}, \boldsymbol{\xi}\right) / \partial \boldsymbol{\xi}$, $\dot{\ell}_{\Gamma}^{(C)}(\boldsymbol{\theta})=\boldsymbol{\Omega}^{-1}(\boldsymbol{S}-\boldsymbol{\Gamma} \boldsymbol{X}) \boldsymbol{X}^{\mathrm{T}}, \dot{\ell}_{\Psi}^{(C)}(\boldsymbol{\theta})=\boldsymbol{\Omega}^{-1}\left\{(\boldsymbol{S}-\boldsymbol{\Gamma} \boldsymbol{X})(\boldsymbol{S}-\boldsymbol{\Gamma} \boldsymbol{X})^{\mathrm{T}}-\boldsymbol{\Omega}\right\} \boldsymbol{\Omega}^{-1} \boldsymbol{\Psi}$, and $\dot{\boldsymbol{\ell}}_{\Sigma}^{(C)}(\boldsymbol{\theta})=\operatorname{diag}\left[\boldsymbol{\Omega}^{-1}\left\{(\boldsymbol{S}-\boldsymbol{\Gamma} \boldsymbol{X})(\boldsymbol{S}-\boldsymbol{\Gamma} \boldsymbol{X})^{\mathrm{T}}-\boldsymbol{\Omega}\right\} \boldsymbol{\Omega}^{-1}\right]$, where $\boldsymbol{\Omega}=\boldsymbol{\Psi} \boldsymbol{\Psi}^{\mathrm{T}}+\boldsymbol{\Sigma}$, and $\operatorname{diag}(\boldsymbol{D})$ is the diagonal matrix that consists of the diagonal elements of $\boldsymbol{D}$. Let

$$
\dot{\boldsymbol{\ell}}_{\theta_{\mathcal{S}}}^{(C)}(\boldsymbol{\theta}) \equiv\left(\dot{\boldsymbol{\ell}}_{\alpha}^{(C)}(\boldsymbol{\theta})^{\mathrm{T}}, \dot{\boldsymbol{\ell}}_{\beta_{\mathcal{S}}}^{(C)}(\boldsymbol{\theta})^{\mathrm{T}}, \dot{\boldsymbol{\ell}}_{\xi}^{(C)}(\boldsymbol{\theta})^{\mathrm{T}}, \operatorname{vec}\left\{\dot{\boldsymbol{\ell}}_{\Gamma}^{(C)}(\boldsymbol{\theta})\right\}^{\mathrm{T}}, \operatorname{vec}\left\{\dot{\boldsymbol{\ell}}_{\Psi}^{(C)}(\boldsymbol{\theta})\right\}^{\mathrm{T}}, \operatorname{vecd}\left\{\dot{\boldsymbol{\ell}}_{\Sigma}^{(C)}(\boldsymbol{\theta})\right\}^{\mathrm{T}}\right)^{\mathrm{T}}
$$

be the vector of the score statistics for a subject with complete data, where $\operatorname{vec}(\boldsymbol{D})$ denotes the vector obtained from stacking the columns of $\boldsymbol{D}$, and $\operatorname{vecd}(\boldsymbol{D})$ denotes the vector of the diagonal elements of $\boldsymbol{D}$. Define $\boldsymbol{V}(\boldsymbol{\alpha}, \boldsymbol{\beta}, \boldsymbol{\xi})=\partial^{2} \log f\left(\boldsymbol{Y} ; \boldsymbol{\alpha}^{\mathrm{T}} \boldsymbol{X}+\right.$ $\left.\boldsymbol{\beta}^{\mathrm{T}} \boldsymbol{S}, \boldsymbol{\xi}\right) / \partial\left(\boldsymbol{\alpha}^{\mathrm{T}}, \boldsymbol{\beta}^{\mathrm{T}}, \boldsymbol{\xi}^{\mathrm{T}}\right)^{\mathrm{T}} \partial\left(\boldsymbol{\alpha}^{\mathrm{T}}, \boldsymbol{\beta}^{\mathrm{T}}, \boldsymbol{\xi}^{\mathrm{T}}\right)$ and $\boldsymbol{I}(\boldsymbol{\theta})=\mathrm{E}\left[\mathrm{E}\left\{\dot{\boldsymbol{\ell}}_{\beta_{\mathcal{N}}}^{(C)}(\boldsymbol{\theta}) \mid \mathcal{O}\right\} \mathrm{E}\left\{\dot{\boldsymbol{\ell}}_{\theta_{\mathcal{S}}}^{(C)}(\boldsymbol{\theta})^{\mathrm{T}} \mid \mathcal{O}\right\}\right]$, where $\mathcal{O}$ denotes the observed data, which consist of $(Y, \boldsymbol{X})$ and a (random) subset of $\boldsymbol{S}$. Let $\boldsymbol{\beta}_{0} \equiv\left(\boldsymbol{\beta}_{0 \mathcal{S}}^{\mathrm{T}}, \boldsymbol{\beta}_{0 \mathcal{N}}^{\mathrm{T}}\right)^{\mathrm{T}} \equiv\left(\beta_{01}, \ldots, \beta_{0 p_{n}}\right)^{\mathrm{T}}$ denote the true value of $\boldsymbol{\beta}$ and $\boldsymbol{\theta}_{0} \equiv$ $\left(\boldsymbol{\alpha}_{0}, \boldsymbol{\beta}_{0}, \boldsymbol{\xi}_{0}, \boldsymbol{\Gamma}_{0}, \boldsymbol{\Psi}_{0}, \boldsymbol{\Sigma}_{0}\right)$ denote the true value of $\boldsymbol{\theta}$. For $k=1, \ldots, K$, let $\boldsymbol{\Psi}_{0}^{(0, k)}$ and $\boldsymbol{\Psi}_{0}^{(k)}$ be the true values of $\Psi^{(0, k)}$ and $\Psi^{(k)}$, respectively. In the sequel, $\|\cdot\|$ denotes the $L_{2}$ norm for vectors or the Frobenius norm for matrices.

We impose the following conditions, some of which involve a generic, finite, and positive constant $C$.

(C1) The vector of covariates $\boldsymbol{X}$ is bounded, and the eigenvalues of $\mathrm{E}\left(\boldsymbol{X} \boldsymbol{X}^{\mathrm{T}}\right)$ lie within $\left(C^{-1}, C\right)$. Also, each component of $\left(\dot{\boldsymbol{\ell}}_{\alpha}^{(C)}\left(\boldsymbol{\theta}_{0}\right), \dot{\boldsymbol{\ell}}_{\beta_{\mathcal{S}}}^{(C)}\left(\boldsymbol{\theta}_{0}\right), \dot{\boldsymbol{\ell}}_{\beta_{\mathcal{N}}}^{(C)}\left(\boldsymbol{\theta}_{0}\right), \dot{\boldsymbol{\ell}}_{\xi}^{(C)}\left(\boldsymbol{\theta}_{0}\right)\right)$ has a finite second moment, and $\lambda_{\min }\left\{-\mathrm{E} \boldsymbol{V}\left(\boldsymbol{\alpha}_{0}, \boldsymbol{\beta}_{0}, \boldsymbol{\xi}_{0}\right)\right\}>C^{-1}$, where $\lambda_{\min }(\boldsymbol{D})$ is the smallest eigenvalue of $\boldsymbol{D}$. In addition, within a small neighborhood of $\left(\boldsymbol{\alpha}_{0}, \boldsymbol{\beta}_{0}, \boldsymbol{\xi}_{0}\right)$ and for any element $v(\boldsymbol{\alpha}, \boldsymbol{\beta}, \boldsymbol{\xi})$ of $\boldsymbol{V}(\boldsymbol{\alpha}, \boldsymbol{\beta}, \boldsymbol{\xi}), v(\boldsymbol{\alpha}, \boldsymbol{\beta}, \boldsymbol{\xi})$ is twice-differentiable with each component of its derivatives up to the second order uniformly bounded by a function 
of $(Y, \boldsymbol{X}, \boldsymbol{S})$ that has a finite second moment.

(C2) The probability $P\left(M_{1}=1, \ldots, M_{p_{n}}=1 \mid Y, \boldsymbol{X}, \overline{\boldsymbol{S}}\right)>C^{-1}$ for almost surely all $(Y, \boldsymbol{X}, \overline{\boldsymbol{S}})$

(C3) The initial estimators satisfy that $\left|\widetilde{\beta}_{j}\right|^{-1}=O_{p}\left(n^{\rho}\right)$ for $j=1, \ldots, p_{1 n}$ and some $\rho \in[0,1 / 2)$ and $\left|\widetilde{\beta}_{j}\right|=O_{p}\left(n^{-\tau}\right)$ for $j=p_{1 n}+1, \ldots, p_{n}$ and some $\tau \in(\kappa, 1 / 2]$.

(C4) The tuning parameter $\lambda_{n}$ satisfies $\lambda_{n} n^{1 / 2+\rho} \rightarrow 0$ as $n \rightarrow \infty$, where $\rho$ is defined in condition (C3).

(C5) The parameters $(\boldsymbol{\Gamma}, \boldsymbol{\Psi}, \boldsymbol{\Sigma})$ satisfy that $\left\|\boldsymbol{\gamma}_{j}\right\|+\left\|\boldsymbol{\psi}_{j}\right\|<C$ and $\sigma_{j}^{2} \in\left(C^{-1}, C\right)$ for $j=1, \ldots, p_{n}$, and the limit of $p_{n}^{-1} \boldsymbol{\Psi}_{0}^{\mathrm{T}} \boldsymbol{\Sigma}_{0}^{-1} \boldsymbol{\Psi}_{0}$ as $n \rightarrow \infty$ exists and has finite and positive eigenvalues. Also, the true values of $\psi_{j j}^{(0,1)}\left(j=1, \ldots, r_{0}\right)$ and $\psi_{j j}^{(k)}(j=$ $\left.1, \ldots, r_{k} ; k=1, \ldots, K\right)$ are bounded below by $C^{-1}$. In addition, for $k=1, \ldots, K$, all eigenvalues of $\widetilde{\boldsymbol{\Psi}}_{0}^{(k) \mathrm{T}} \widetilde{\boldsymbol{\Psi}}_{0}^{(k)}$ are bounded below by $C^{-1}$, where $\widetilde{\boldsymbol{\Psi}}_{0}^{(k)} \equiv\left(\boldsymbol{\Psi}_{0}^{(0, k)}, \mathbf{\Psi}_{0}^{(k)}\right)$.

(C6) Let $\boldsymbol{S}^{(O)}$ be an arbitrary subvector of $\boldsymbol{S}, \boldsymbol{\nu}=(\boldsymbol{\alpha}, \boldsymbol{\beta}, \boldsymbol{\xi}), \nu_{j}$ be the $j$ th component of $\boldsymbol{\nu}\left(j=1, \ldots, q_{n}\right)$, and $q_{n}$ be the dimension of $\boldsymbol{\nu}$. Let $\mathcal{H}_{1}$ and $\mathcal{H}_{2}$ be classes of functions defined as

$$
\begin{gathered}
\mathcal{H}_{1}=\left\{h:(Y, \boldsymbol{X}, \boldsymbol{S}, \boldsymbol{\theta}) \mapsto \frac{\partial^{2}}{\partial \nu_{j} \partial \nu_{k}} \log f\left(Y ; \boldsymbol{\alpha}^{\mathrm{T}} \boldsymbol{X}+\boldsymbol{\beta}^{\mathrm{T}} \boldsymbol{S}, \boldsymbol{\xi}\right) ; j, k=1, \ldots, q_{n}\right\}, \\
\mathcal{H}_{2}=\left\{h:(Y, \boldsymbol{X}, \boldsymbol{S}, \boldsymbol{\theta}) \mapsto\left\{\frac{\partial}{\partial \nu_{j}} \log f\left(Y ; \boldsymbol{\alpha}^{\mathrm{T}} \boldsymbol{X}+\boldsymbol{\beta}^{\mathrm{T}} \boldsymbol{S}, \boldsymbol{\xi}\right)\right\}^{k_{0}} \prod_{h=1}^{p_{n}} S_{h}^{k_{h}} ; j=1, \ldots, q_{n} ;\right. \\
\left.k_{0}=0,1, \text { or } 2 ;\left(k_{1}, \ldots, k_{p_{n}}\right) \text { are nonnegative integers; } \sum_{h=1}^{p_{n}} k_{h} \leq 4\right\},
\end{gathered}
$$

and $\mathcal{H}=\mathcal{H}_{1} \cup \mathcal{H}_{2}$. Let $f\left(\boldsymbol{S} \mid Y, \boldsymbol{X}, \boldsymbol{S}^{(O)} ; \boldsymbol{\theta}\right)$ be the conditional density function of $\boldsymbol{S}$ given $\left(Y, \boldsymbol{X}, \boldsymbol{S}^{(O)}\right), \dot{\boldsymbol{f}}\left(\boldsymbol{S} \mid Y, \boldsymbol{X}, \boldsymbol{S}^{(O)} ; \boldsymbol{\theta}\right)=\partial f\left(\boldsymbol{S} \mid Y, \boldsymbol{X}, \boldsymbol{S}^{(O)} ; \boldsymbol{\theta}\right) / \partial \boldsymbol{\theta}$, and $\boldsymbol{S}^{(M)}$ 
be the vector of components of $\boldsymbol{S}$ that are not in $\boldsymbol{S}^{(O)}$. For $\widetilde{\boldsymbol{\theta}}$ and $\boldsymbol{\theta}$ within a small neighborhood of $\boldsymbol{\theta}_{0}$ and for all $h \in \mathcal{H}$, each component of

$$
\mathrm{E} \int h\left(Y, \boldsymbol{X}, \boldsymbol{S}^{(O)}, \widetilde{\boldsymbol{\theta}}\right) \dot{\boldsymbol{f}}\left(\boldsymbol{S} \mid Y, \boldsymbol{X}, \boldsymbol{S}^{(O)} ; \boldsymbol{\theta}\right) \mathrm{d} \boldsymbol{S}^{(M)}
$$

is bounded by $C$, and $\mathrm{E}\left\{h(Y, \boldsymbol{X}, \boldsymbol{S}, \boldsymbol{\theta}) \mid Y, \boldsymbol{X}, \boldsymbol{S}^{(O)} ; \boldsymbol{\theta}\right\}$ is four-times differentiable with respect to $\boldsymbol{\theta}$ with each component of its derivatives up to the fourth order uniformly bounded by a function of $\left(Y, \boldsymbol{X}, \boldsymbol{S}^{(O)}\right)$ that has a finite second moment.

(C7) The score statistics for the outcome model satisfy $\mathrm{E}\left|Z\left(\boldsymbol{\alpha}_{0}^{\mathrm{T}} \boldsymbol{X}+\boldsymbol{\beta}_{0}^{\mathrm{T}} \boldsymbol{S}, \boldsymbol{\xi}_{0}\right) S_{j}\right|^{k} \leq k ! C^{k}$ for $j=p_{1 n}+1, \ldots, p_{n}$ and $k \geq 2$.

(C8) For some $\eta \in(1-\tau, 1-\kappa)$,

$$
\sup _{\boldsymbol{\theta}}\|\boldsymbol{I}(\boldsymbol{\theta})\|_{2, \infty}=O\left(n^{\tau+\eta-1}\right)
$$

where $\|\boldsymbol{D}\|_{2, \infty}=\left\|\sup _{\|\boldsymbol{v}\|=1} \boldsymbol{D} \boldsymbol{v}\right\|_{\infty}$, the supremum is taken in a small neighborhood of $\boldsymbol{\theta}_{0}$, and $\tau$ is defined in condition (C3).

(C9) The tuning parameter $\lambda_{n}$ satisfies $\lambda_{n} n^{3 / 2-\kappa-\eta} \rightarrow \infty$, where $\eta$ is chosen in condition (C8).

Remark 1. Condition (C1) pertains to regularity conditions on the outcome model and guarantees that with complete data, a local maximizer of the log-likelihood function is consistent for $(\boldsymbol{\alpha}, \boldsymbol{\beta}, \boldsymbol{\xi})$. Condition (C2) requires that a nonvanishing proportion of subjects have complete data. Condition (C3) requires that the initial estimators of the nonzero parameters do not tend to zero at a rate faster than $n^{-\rho}$, whereas the estimators of the zero parameters are $n^{\tau}$-consistent. This condition implies that the signal strength 


\section{ASYMPTOTIC PROPERTIES OF THE PENALIZED ESTIMATORS}

of $S_{j}\left(j=1, \ldots, p_{1 n}\right)$ is bounded below by $C n^{-\rho}$ for some positive constant $C$. Condition (C5) pertains to regularity conditions for consistent estimation of the latent factor model, and condition (C6) pertains to regularity conditions on the conditional density function of the missing data given the observed data. Condition $(\mathrm{C} 7)$ pertains to high-order moments of the score statistics of the outcome model, and condition (C8) is a general and weaker version of the strong irrepresentable condition (Zhao and $\mathrm{Yu}, 2006)$; these two conditions are imposed to ensure consistent model selection. Conditions (C4) and (C9) jointly require that $n^{1 / 2+\rho} \ll \lambda_{n}^{-1} \ll n^{3 / 2-\kappa-\eta}$. They ensure that the penalty for $\boldsymbol{\beta}$ is strong enough to impose model sparsity but weak enough to yield consistent estimation of the nonzero parameters.

Remark 2. If we set the marginal estimators described in Section 2 as the initial estimators, then condition (C3) pertains to the relationships among the factor loadings across different features. Note that the marginal estimators are the MLE under the full likelihood (that incorporates the distribution of the incomplete variables) and thus are $n^{1 / 2}$-consistent for the "true" marginal regression parameters. In light of Proposition 1 of Fan and Song (2010), we can show that a marginal regression parameter under a generalized linear model of $Y$ tends to zero at a certain rate if and only if the correlation between the corresponding feature and $\boldsymbol{S}_{\mathcal{S}}^{\mathrm{T}} \boldsymbol{\beta}_{0 \mathcal{S}}$ tends to zero at the same rate. Therefore, condition (C3) holds if $\left|\sum_{j=1}^{p_{1 n}} \beta_{0 j} \boldsymbol{\psi}_{0 j}^{\mathrm{T}} \boldsymbol{\psi}_{0 k}+\beta_{0 k} \sigma_{0 k}^{2}\right|>C n^{-\rho}$ for $k=1, \ldots, p_{1 n}$ and some positive constant $C$ and $\sum_{j=1}^{p_{1 n}} \beta_{0 j} \psi_{0 j}^{\mathrm{T}} \boldsymbol{\psi}_{0 k}=O\left(n^{-\tau}\right)$ for $k=p_{1 n}+1, \ldots, p_{n}$, where for $j=1, \ldots, p_{n}, \boldsymbol{\psi}_{0 j}^{\mathrm{T}}$ is the $j$ th row of $\boldsymbol{\Psi}_{0}$, and $\sigma_{0 j}^{2}$ is the $j$ th diagonal element of $\boldsymbol{\Sigma}_{0}$.

Let $\boldsymbol{H}$ be the projection matrix onto the linear space of $\boldsymbol{\Sigma}_{0}^{-1 / 2} \boldsymbol{\Psi}_{0}$. Our main theoretical 


\section{ASYMPTOTIC PROPERTIES OF THE PENALIZED ESTIMATORS}

results are summarized in the following theorem, whose proof is given in Appendix A.2.

Theorem 1. Under conditions $(C 1)-(C 9)$, a local maximizer of $p \ell_{n}(\boldsymbol{\theta})$, denoted by $\widehat{\boldsymbol{\theta}} \equiv$ $\left(\widehat{\boldsymbol{\alpha}}, \widehat{\boldsymbol{\beta}}_{\mathcal{S}}, \widehat{\boldsymbol{\beta}}_{\mathcal{N}}, \widehat{\boldsymbol{\xi}}, \widehat{\boldsymbol{\Gamma}}, \widehat{\boldsymbol{\Psi}}, \widehat{\boldsymbol{\Sigma}}\right)$, satisfies that

1. $\left\|\widehat{\boldsymbol{\alpha}}-\boldsymbol{\alpha}_{0}\right\|+\left\|\widehat{\boldsymbol{\beta}}_{\mathcal{S}}-\boldsymbol{\beta}_{0 \mathcal{S}}\right\|+\left\|\widehat{\boldsymbol{\xi}}-\boldsymbol{\xi}_{0}\right\|+\left\|(\boldsymbol{I}-\boldsymbol{H}) \boldsymbol{\Sigma}_{0}^{-1 / 2}\left(\widehat{\boldsymbol{\Gamma}}-\boldsymbol{\Gamma}_{0}\right)\right\|+\|(\boldsymbol{I}-\boldsymbol{H}) \boldsymbol{\Sigma}_{0}^{-1 / 2}(\widehat{\boldsymbol{\Psi}}-$ $\left.\boldsymbol{\Psi}_{0}\right)\|+\| \widehat{\boldsymbol{\Sigma}}-\boldsymbol{\Sigma}_{0} \|=O_{p}\left(n^{-1 / 2} p_{n}^{1 / 2}\right) ;$

2. $\left\|\boldsymbol{H} \boldsymbol{\Sigma}_{0}^{-1 / 2}\left(\widehat{\boldsymbol{\Gamma}}-\boldsymbol{\Gamma}_{0}\right)\right\|+\left\|\boldsymbol{H} \boldsymbol{\Sigma}_{0}^{-1 / 2}\left(\widehat{\boldsymbol{\Psi}}-\mathbf{\Psi}_{0}\right)\right\|=O_{p}\left(n^{-1 / 2} p_{n}\right)$; and

3. $P\left(\widehat{\boldsymbol{\beta}}_{\mathcal{N}}=\mathbf{0}\right) \rightarrow 1$ as $n \rightarrow \infty$.

Remark 3. This theorem provides the rate of convergence for the estimators of the nonzero parameters and states that the estimators of the zero parameters are equal to zero with probability tending to 1 . A major step in the proof of Theorem 1 is to construct a shrinking neighborhood $\mathcal{N}$ of the true parameter values and show that $p \ell_{n}\left(\boldsymbol{\theta}_{0}\right)>\sup _{\boldsymbol{\theta} \in \partial \mathcal{N}} p \ell_{n}(\boldsymbol{\theta})$ with probability tending to 1 , where $\partial \mathcal{N}$ denotes the boundary of $\mathcal{N}$; similar approaches were adopted by Fan and Li (2001) and Fan and Peng (2004) to prove the consistency of the smoothly clipped absolute deviation estimator. The proof is substantially more difficult for the factor model than for conventional regression models, because the largest eigenvalues of $\left(\boldsymbol{\Psi} \boldsymbol{\Psi}^{\mathrm{T}}+\boldsymbol{\Sigma}\right)$ diverge to infinity (Bai and Liao, 2016). A key innovation in our proof is to identify the few eigenvalues of the Hessian matrix of $p \ell_{n}(\cdot)$ that tend to 0 (as a result of the unboundedness of the eigenvalues of $\left(\boldsymbol{\Psi} \boldsymbol{\Psi}^{\mathrm{T}}+\boldsymbol{\Sigma}\right)$ ) and construct an "elliptical" $\mathcal{N}$ with diameter of order $n^{-1 / 2} p_{n}$ in directions that correspond to their eigenvectors and with diameter of order $n^{-1 / 2} p_{n}^{1 / 2}$ in other directions. This construction guarantees that the second-order term in the Taylor series expansion 


\section{ASYMPTOTIC PROPERTIES OF THE PENALIZED ESTIMATORS}

of $p \ell_{n}(\boldsymbol{\theta})$ at $\boldsymbol{\theta}_{0}$ is negative and dominates the first-order term at any $\boldsymbol{\theta} \in \partial \mathcal{N}$ with probability tending to 1 . Using this construction, we prove that the projections of $\boldsymbol{\Sigma}_{0}^{-1 / 2} \widehat{\boldsymbol{\Gamma}}$ and $\boldsymbol{\Sigma}_{0}^{-1 / 2} \widehat{\boldsymbol{\Psi}}$ along the linear space of $\boldsymbol{\Sigma}_{0}^{-1 / 2} \boldsymbol{\Psi}_{0}$ are consistent at the $\left(n^{1 / 2} p_{n}^{-1}\right)$-rate, whereas the estimators of all other nonzero parameters, including the regression parameters of interest, are consistent at the conventional $\left(n^{1 / 2} p_{n}^{-1 / 2}\right)$-rate.

Remark 4. Bai and Li (2012) proved that the MLE of an unstructured factor loading matrix $\Psi$ with complete data is $n^{-1 / 2} p_{n}^{1 / 2}$-consistent; this rate is faster than that given in Theorem 1. However, the arguments of Bai and Li (2012) are not applicable to our setting, especially when the missing-data mechanism depends on $\boldsymbol{S}$ (through $Y$ ). The asymptotic properties of the MLE of the factor model in the presence of missing data have not been previously studied, and it is unclear whether the convergence rates given in Theorem 1 can be improved.

Remark 5. The dimension of the features, $p_{n}$, that we allow is smaller than that in the existing work on penalized regression with complete data (Fan and Peng, 2004, Fan and Lv, 2011) for three reasons. First, because the likelihood involves the model of the incomplete variables, the number of (nonzero) parameters is much larger than that of the existing work under the same number of features. Second, the rate of convergence of the proposed estimators is slower than that of the existing work, such that a smaller $p_{n}$ is required for the consistency of certain functions of the parameters, such as the information matrix. Third, due to the structure of the factor model, the sizes of the derivatives of the log-likelihood function are potentially larger than those in conventional regression models, such that a smaller $p_{n}$ is required to guarantee the concavity of the (observed) 
log-likelihood function around the true parameter values.

\section{Simulation studies}

We considered two types of features, $\boldsymbol{S}^{(1)}$ and $\boldsymbol{S}^{(2)}$, both with dimension $p / 2$. We generated the features from the following factor model:

$$
\boldsymbol{S}^{(k)}=\boldsymbol{\psi}^{(0, k)} U^{(0)}+\boldsymbol{\psi}^{(k)} U^{(k)}+\boldsymbol{\epsilon}^{(k)} \text { for } k=1,2,
$$

where $U^{(0)}, U^{(1)}$, and $U^{(2)}$ are independent standard normal variables, $\boldsymbol{\epsilon}^{(1)}$ and $\boldsymbol{\epsilon}^{(2)}$ are independent $(p / 2)$-variate standard normal variables, and $\boldsymbol{\psi}^{(0,1)}, \boldsymbol{\psi}^{(0,2)}, \boldsymbol{\psi}^{(1)}$, and $\boldsymbol{\psi}^{(2)}$ are $(p / 2)$-vectors of factor loadings. We set

$$
\begin{gathered}
\boldsymbol{\psi}^{(0,1)}=\boldsymbol{\psi}^{(0,2)}=(\underbrace{0.2, \ldots, 0.2}_{20 \text { terms }}, \underbrace{-0.2, \ldots,-0.2}_{(p / 4-10) \text { terms }}, \underbrace{0.2, \ldots, 0.2}_{(p / 4-10) \text { terms }})^{\mathrm{T}}, \\
\boldsymbol{\psi}^{(1)}=\boldsymbol{\psi}^{(2)}=(\underbrace{0.4, \ldots, 0.4}_{20 \text { terms }}, \underbrace{0.4, \ldots, 0.4}_{(p / 4-10) \text { terms }}, \underbrace{-0.2, \ldots,-0.2}_{(p / 4-10) \text { terms }})^{\mathrm{T}} .
\end{gathered}
$$

In this setting, the first 20 components of each type of features are positively associated with both the common and type-specific latent variables, whereas the remaining components are negatively associated with either the common or type-specific latent variable. As a result, the first 20 components of each type of features are relatively strongly associated with each other, but they are weakly associated with other features. We let the outcome variable $Y$ be continuous or binary. For the continuous case, we set $Y=\sum_{j=1}^{15} 0.05\left(S_{j}^{(1)}+S_{j}^{(2)}\right)+\delta$, where $\delta$ follows a standard normal distribution, and $S_{j}^{(k)}$ is the $j$ th component of $\boldsymbol{S}^{(k)}(k=1,2)$. For the binary case, we set $P\left(Y=1 \mid \boldsymbol{S}^{(1)}, \boldsymbol{S}^{(2)}\right)=\operatorname{logit}^{-1}\left\{-3+\sum_{j=1}^{15} 0.15\left(S_{j}^{(1)}+S_{j}^{(2)}\right)\right\}$, such that $P(Y=1) \approx 0.1$. We set $\boldsymbol{S}^{(1)}$ to be completely observed and $\boldsymbol{S}^{(2)}$ to be missing for $50 \%$ of the subjects 
based on one of the following missing-data mechanisms: (1) missing completely at random (MCAR); and (2) missing at random (MAR), such that in the case of the continuous outcome variable, $\boldsymbol{S}^{(2)}$ is observed for subjects with extreme values of $Y$, and in the case of the binary outcome variable, $\boldsymbol{S}^{(2)}$ is observed for all subjects with $Y=1$ and for a random subset of subjects with $Y=0$.

We adopted two penalization methods: lasso (Tibshirani, 1996) and adaptive lasso (Zou, 2006). For lasso, we set each weight term $w_{j}$ to be 1 . For adaptive lasso, we used the MLE of the regression parameters in the marginal regression models of $Y$ against $S_{j}^{(k)}(j=$ $1, \ldots, p / 2 ; k=1,2)$ as the initial estimators. The tuning parameter for each method was selected by 5 -fold cross-validation, where the cross-validation error is defined as the negative log-likelihood value, and the grid for $\lambda$ is $\left\{(0.01)^{j / 100} \lambda_{\max }\right\}_{j=0,1, \ldots, 99}$. For each penalization method, we considered four methods for handling missing data: (1) variable selection on complete cases only; (2) variable selection with missing data (singly) imputed using the structured matrix completion method of Cai, Cai and Zhang (2016), where the row thresholding parameter is set to be $2\left\{p / \operatorname{length}\left(\boldsymbol{S}^{(2)}\right)\right\}^{1 / 2} \approx 2.828$; (3) variable selection with missing data imputed by the posterior expectation under the proposed factor model, with the factor model estimated using only $\left(\boldsymbol{S}^{(1)}, \boldsymbol{S}^{(2)}\right)$; and (4) the proposed penalizedlikelihood method. For method (4) and the estimation of the factor model in method (3), we used the function GQN2_ORDER of the C++ software SPARSE_GRID_HW (available at https://people.sc.fsu.edu/ jburkardt/cpp_src/sparse_grid_hw/sparse_grid_hw.html) and set level $=3$ to generate the nodes and weights for the sparse-grid quadrature. We terminated the EM algorithm when the maximum absolute difference between the parameter estimators of two consecutive iterations became smaller than $10^{-5}$. For methods (3) and 
(4), we considered models with $\left(r_{0}, r_{1}, r_{2}\right) \in\left\{\left(r_{0}, r_{1}, r_{2}\right): \sum_{k=0}^{2}\left|r_{k}-1\right| \leq 1, r_{0} \neq 0\right\}$ and selected the model using the Bayesian information criterion (BIC) (Schwarz, 1978). Note that the models considered differ from the true model by at most one latent variable. Models with $r_{0}=0$ assume independence between the two types of features and thus were not considered. In all models, we set $X=1$.

We set $n=500$ and $p=100$ or 300 . For each method, we report the number of variables selected, false discovery rate, true positive rate, and prediction error. The false discovery rate is defined as the proportion of selected variables that have zero true parameter value, and the true positive rate is defined as the proportion of variables with nonzero true parameter values that are selected. Prediction error is defined as $\mathrm{E}\left\{\boldsymbol{S}^{(1) \mathrm{T}}\left(\widehat{\boldsymbol{\beta}}^{(1)}-\boldsymbol{\beta}_{0}^{(1)}\right)+\boldsymbol{S}^{(2) \mathrm{T}}\left(\widehat{\boldsymbol{\beta}}^{(2)}-\boldsymbol{\beta}_{0}^{(2)}\right)\right\}^{2}$, where $\widehat{\boldsymbol{\beta}}^{(k)}$ and $\boldsymbol{\beta}_{0}^{(k)}$ are the estimated and true values of the regression parameters of $\boldsymbol{S}^{(k)}(k=1,2)$, respectively. For the single imputation method based on the factor model and the proposed method, we also report the proportion of replicates in which the correct numbers of latent variables are selected. The results, which are based on 200 replicates, are summarized in Tables 1 and 2 .

With the sparse-grid numerical integration, the mean computing time for a single E-step is about $0.1-0.2$ seconds under $p=100$ and about $0.3-0.7$ seconds under $p=$ 300 in various settings. In all scenarios, the proposed method performs substantially better than the complete-case analysis, because the latter discards subjects with partial information and thus is less efficient. Single imputation based on the factor model has overall better variable selection and prediction performance than single imputation based on structured matrix completion, because the former assumes a correct imputation model. Under MCAR, the proposed method and the single imputation method based on the 
Table 1: Simulation results for the continuous outcome variable

\begin{tabular}{|c|c|c|c|c|c|c|c|c|c|c|}
\hline & \multicolumn{5}{|c|}{ Lasso } & \multicolumn{5}{|c|}{ A-Lasso } \\
\hline & $\begin{array}{l}\text { Variables } \\
\text { selected }\end{array}$ & FDR & TPR & $\begin{array}{l}\text { Pred } \\
\text { error }\end{array}$ & $\begin{array}{c}\text { Correct } \\
\text { model }\end{array}$ & $\begin{array}{c}\text { Variables } \\
\text { selected }\end{array}$ & FDR & TPR & $\begin{array}{l}\text { Pred } \\
\text { error }\end{array}$ & $\begin{array}{c}\text { Correct } \\
\text { model }\end{array}$ \\
\hline \multicolumn{11}{|l|}{$\mathrm{MCAR} ; p=100$} \\
\hline Complete & 26.4 & 0.372 & 0.531 & 0.125 & $\mathrm{~N} / \mathrm{A}$ & 22.4 & 0.280 & 0.526 & 0.122 & $\mathrm{~N} / \mathrm{A}$ \\
\hline $\mathrm{SMC}$ & 28.9 & 0.377 & 0.584 & 0.120 & $\mathrm{~N} / \mathrm{A}$ & 23.7 & 0.276 & 0.555 & 0.122 & $\mathrm{~N} / \mathrm{A}$ \\
\hline Imputed & 30.2 & 0.354 & 0.632 & 0.098 & 1 & 25.0 & 0.243 & 0.616 & 0.096 & 1 \\
\hline Proposed & 25.6 & 0.294 & 0.592 & 0.105 & 1 & 23.0 & 0.218 & 0.591 & 0.098 & 1 \\
\hline \multicolumn{11}{|l|}{$\mathrm{MAR} ; p=100$} \\
\hline Complete & 32.0 & 0.350 & 0.670 & 0.160 & $\mathrm{~N} / \mathrm{A}$ & 26.3 & 0.231 & 0.661 & 0.206 & $\mathrm{~N} / \mathrm{A}$ \\
\hline $\mathrm{SMC}$ & 28.8 & 0.355 & 0.589 & 0.150 & $\mathrm{~N} / \mathrm{A}$ & 22.2 & 0.203 & 0.570 & 0.152 & $\mathrm{~N} / \mathrm{A}$ \\
\hline Imputed & 31.7 & 0.371 & 0.644 & 0.134 & 1 & 25.3 & 0.239 & 0.629 & 0.149 & 1 \\
\hline Proposed & 27.9 & 0.269 & 0.668 & 0.089 & 1 & 24.5 & 0.186 & 0.654 & 0.080 & 1 \\
\hline \multicolumn{11}{|l|}{$\mathrm{MCAR} ; p=300$} \\
\hline Complete & 29.9 & 0.497 & 0.454 & 0.156 & $\mathrm{~N} / \mathrm{A}$ & 35.6 & 0.533 & 0.507 & 0.168 & $\mathrm{~N} / \mathrm{A}$ \\
\hline $\mathrm{SMC}$ & 34.3 & 0.485 & 0.547 & 0.130 & $\mathrm{~N} / \mathrm{A}$ & 32.4 & 0.428 & 0.577 & 0.123 & $\mathrm{~N} / \mathrm{A}$ \\
\hline Imputed & 34.0 & 0.462 & 0.567 & 0.124 & 0.910 & 36.9 & 0.465 & 0.607 & 0.130 & 0.910 \\
\hline Proposed & 24.2 & 0.366 & 0.495 & 0.140 & 0.910 & 27.6 & 0.367 & 0.556 & 0.120 & 0.915 \\
\hline \multicolumn{11}{|l|}{$\mathrm{MAR} ; p=300$} \\
\hline Complete & 36.9 & 0.473 & 0.600 & 0.153 & $\mathrm{~N} / \mathrm{A}$ & 34.2 & 0.412 & 0.626 & 0.236 & $\mathrm{~N} / \mathrm{A}$ \\
\hline $\mathrm{SMC}$ & 40.1 & 0.504 & 0.616 & 0.157 & $\mathrm{~N} / \mathrm{A}$ & 35.9 & 0.408 & 0.649 & 0.198 & $\mathrm{~N} / \mathrm{A}$ \\
\hline Imputed & 34.0 & 0.462 & 0.568 & 0.151 & 0.995 & 34.4 & 0.427 & 0.615 & 0.189 & 0.995 \\
\hline Proposed & 26.5 & 0.320 & 0.586 & 0.118 & 0.995 & 27.2 & 0.294 & 0.615 & 0.094 & 0.995 \\
\hline
\end{tabular}

NOTE: "A-Lasso" stands for adaptive lasso; "Complete," "SMC," "Imputed," and "Proposed" stand for the complete-case analysis, the structured matrix completion method of Cai, Cai and Zhang (2016), single imputation based on the factor model, and the proposed method, respectively; "Variables selected," "FDR," "TPR," "Pred error," and "Correct model" stand for the average number of variables selected, the false discovery rate, the true positive rate, the prediction error, and the proportion of replicates in which the correct factor model is selected, respectively. 
Table 2: Simulation results for the binary outcome variable

\begin{tabular}{|c|c|c|c|c|c|c|c|c|c|c|}
\hline & \multicolumn{5}{|c|}{ Lasso } & \multicolumn{4}{|c|}{ A-Lasso } & \multirow{2}{*}{$\begin{array}{c}\text { Correct } \\
\text { model }\end{array}$} \\
\hline & $\begin{array}{c}\text { Variables } \\
\text { selected }\end{array}$ & FDR & TPR & $\begin{array}{l}\text { Pred } \\
\text { error }\end{array}$ & $\begin{array}{c}\text { Correct } \\
\text { model }\end{array}$ & $\begin{array}{c}\text { Variables } \\
\text { selected }\end{array}$ & FDR & TPR & $\begin{array}{l}\text { Pred } \\
\text { error }\end{array}$ & \\
\hline \multicolumn{11}{|c|}{$\mathrm{MCAR} ; p=100$} \\
\hline Complete & 20.0 & 0.356 & 0.408 & 1.486 & $\mathrm{~N} / \mathrm{A}$ & 17.1 & 0.286 & 0.396 & 1.507 & $\mathrm{~N} / \mathrm{A}$ \\
\hline SMC & 23.2 & 0.364 & 0.468 & 1.451 & $\mathrm{~N} / \mathrm{A}$ & 18.8 & 0.274 & 0.439 & 1.370 & $\mathrm{~N} / \mathrm{A}$ \\
\hline Imputed & 24.8 & 0.343 & 0.522 & 1.214 & 1 & 20.7 & 0.251 & 0.504 & 1.113 & 1 \\
\hline Proposed & 24.4 & 0.353 & 0.506 & 1.277 & 1 & 20.5 & 0.251 & 0.502 & 1.100 & 1 \\
\hline \multicolumn{11}{|c|}{ MAR; $p=100$} \\
\hline Complete & 25.8 & 0.340 & 0.550 & 1.134 & $\mathrm{~N} / \mathrm{A}$ & 20.4 & 0.227 & 0.515 & 1.075 & $\mathrm{~N} / \mathrm{A}$ \\
\hline $\mathrm{SMC}$ & 23.7 & 0.338 & 0.498 & 1.464 & $\mathrm{~N} / \mathrm{A}$ & 19.0 & 0.220 & 0.481 & 1.530 & $\mathrm{~N} / \mathrm{A}$ \\
\hline Imputed & 25.0 & 0.337 & 0.534 & 1.272 & 1 & 20.5 & 0.232 & 0.513 & 1.430 & 1 \\
\hline Proposed & 27.2 & 0.341 & 0.577 & 1.101 & 1 & 21.8 & 0.227 & 0.552 & 0.966 & 1 \\
\hline \multicolumn{11}{|c|}{$\mathrm{MCAR} ; p=300$} \\
\hline Complete & 23.4 & 0.508 & 0.338 & 1.827 & $\mathrm{~N} / \mathrm{A}$ & 26.8 & 0.535 & 0.388 & 2.300 & $\mathrm{~N} / \mathrm{A}$ \\
\hline $\mathrm{SMC}$ & 28.4 & 0.495 & 0.432 & 1.592 & $\mathrm{~N} / \mathrm{A}$ & 27.6 & 0.462 & 0.460 & 1.462 & $\mathrm{~N} / \mathrm{A}$ \\
\hline Imputed & 29.1 & 0.488 & 0.452 & 1.515 & 0.915 & 30.4 & 0.478 & 0.490 & 1.488 & 0.915 \\
\hline Proposed & 27.5 & 0.487 & 0.426 & 1.624 & 0.880 & 30.7 & 0.486 & 0.484 & 1.389 & 0.910 \\
\hline \multicolumn{11}{|c|}{$\mathrm{MAR} ; p=300$} \\
\hline Complete & 30.9 & 0.498 & 0.484 & 1.371 & $\mathrm{~N} / \mathrm{A}$ & 27.4 & 0.422 & 0.499 & 1.379 & $\mathrm{~N} / \mathrm{A}$ \\
\hline $\mathrm{SMC}$ & 31.4 & 0.488 & 0.494 & 1.502 & $\mathrm{~N} / \mathrm{A}$ & 28.1 & 0.409 & 0.523 & 1.975 & $\mathrm{~N} / \mathrm{A}$ \\
\hline Imputed & 25.7 & 0.440 & 0.446 & 1.531 & 0.990 & 27.3 & 0.425 & 0.491 & 2.003 & 0.990 \\
\hline Proposed & 31.5 & 0.479 & 0.511 & 1.346 & 0.990 & 30.8 & 0.443 & 0.540 & 1.182 & 0.990 \\
\hline
\end{tabular}

NOTE: See NOTE to Table 1 
factor model perform similarly, possibly because the structure of the factor model can be accurately recovered using $\left(\boldsymbol{S}^{(1)}, \boldsymbol{S}^{(2)}\right)$ alone. Under MAR, however, the proposed method yields substantially smaller prediction error and similar or better false discovery and true positive rates than the single imputation methods, due to either the estimation bias of the factor model or the failure to recover the low-rank structure underlying $\left(\boldsymbol{S}^{(1)}, \boldsymbol{S}^{(2)}\right)$ in the single imputation methods. Note that the proposed method generally yields better results under MAR than under MCAR, because subjects with extreme outcome values contain more information than randomly selected subjects. Due to estimation bias, however, the single imputation methods fail to capture the extra information and perform worse under MAR. For the single imputation method based on the factor model and the proposed method, BIC selects the correct factor model in the majority of replicates.

Adaptive lasso generally yields lower false discovery rate and smaller prediction error than lasso; in some cases, lasso yields higher true positive rate than adaptive lasso, probably because lasso selects more variables. These results agree with the expectation that by assigning larger penalties to less important variables, adaptive lasso outperforms lasso.

We conducted additional simulation studies to investigate the performance of the proposed methods under a misspecified latent factor model. We showed that when every feature depends on all three latent variables, the proposed method with $r_{0}=r_{1}=r_{2}=1$ still yields superior performance over the complete-case analysis and imputation methods. Details of the simulation settings and results are presented in Section S1 of the supplementary materials. 


\section{A real study}

We considered the TCGA data (available at http://gdac.broadinstitute.org/) on two smoking-related, upper aerodigestive tract cancers: head and neck squamous cell carcinoma (HNSC) and lung adenocarcinoma (LUAD). After removing patients with missing clinical data, the total sample size was 955, with 448 HNSC patients and 507 LUAD patients. We considered the outcome variable tumor stage, dichotomized into stage I/II and stage III/IV. The proportions of patients with later stages were 0.77 for HNSC and 0.22 for LUAD. We considered two types of genomic features, gene expressions and protein expressions, with 18028 and 155 variables, respectively. A total of 400 patients had no protein data due to insufficient tissue sample left for protein expression measurement, so the missing mechanism does not depend on the protein expression values. In addition, patients missed 763 gene expressions on average, and among patients with some protein data, an average of 4.3 protein expressions are missing; the missing data did not exhibit a blockwise pattern. The missing-data pattern is plotted in Figure S1 in the supplementary materials.

We first screened the gene expressions according to their marginal associations with tumor stage, such that the resulting number of variables and sample size were comparable. We tested each gene expression's marginal association with tumor stage using the score test (adjusted for cancer type), with the model for missing data included in the likelihood, and we selected the 500 variables with the smallest $p$-values. Then, we fit a logistic regression model for tumor stage, with cancer type as $X$ and the screened gene expressions and protein expressions as $\boldsymbol{S}$. For the latent factor model, we set cancer type as a 


\section{A REAL STUDY}

covariate and ranged the total number of latent variables $r$ from 3 to 6 with $\left(r_{0}, r_{1}, r_{2}\right) \in$ $\left\{\left(r_{0}, r_{1}, r_{2}\right): \sum_{k=0}^{2} r_{k} \leq 6, r_{k} \geq 1\right.$ for $\left.k=0,1,2\right\}$. We used the adaptive-lasso penalty with the marginal regression parameter estimators as the initial estimators and selected the tuning parameter using 5 -fold cross validation.

The BIC picked $\left(r_{0}, r_{1}, r_{2}\right)=(2,3,1)$ for the factor model. A total of 53 genomic features were selected, with 45 gene expressions and 8 protein expressions; the selected features are presented in Table S3 in the supplementary materials. Several selected genes, including WDR37, FUT7, and DDIT4, were previously reported to be associated with metastasis or patient survival (Ogawa, Inoue and Koide, 1997; Läubli et al., 2006; Wang et al. 2015). The selected proteins include the epidermal growth factor receptor, which is known to be involved in the pathogenesis and progression of different types of cancer (Nicholson, Gee and Harper, 2001; Normanno et al., 2006).

The estimated outcome model and factor model enabled us to construct a personal genomic risk score. For each patient, we calculated the posterior expectation of the latent variable, denoted by $\widehat{\boldsymbol{U}}$, and imputed the missing values of the genomic features by the corresponding element of $\boldsymbol{\Gamma} \boldsymbol{X}+\boldsymbol{\Psi} \widehat{\boldsymbol{U}}$, where the parameters were evaluated at their estimated values. The risk score is defined as $\boldsymbol{\beta}^{\mathrm{T}} \widehat{\boldsymbol{S}}$, with $\boldsymbol{\beta}$ evaluated at its estimated value and $\widehat{\boldsymbol{S}}$ the vector of the observed or imputed genomic features.

We evaluated the association between the risk score and progression-free survival time (since initial diagnosis). For each cancer type, we fit a stratified Cox model of progressionfree survival time against the risk score, stratified by tumor stage. The likelihood-ratio $p$-values for the effects of the risk score are $4.20 \times 10^{-4}$ and $6.41 \times 10^{-6}$ for HNSC and LUAD, respectively. These $p$-values are highly significant, suggesting that the selected 
genomic features are highly relevant to cancer progression. Note that the results are not due to overfitting, because progression-free survival time is not involved in the calculation of the risk score, and all evaluations are stratified by tumor stage and cancer type.

For comparisons, we performed similar analyses using imputed data and the complete cases only. (The method developed by Cai, Cai and Zhang (2016) is not applicable to the general missing-data pattern exhibited in this data set.) For single imputation, we imputed the missing values using $k$-nearest-neighbor imputation (Troyanskaya et al., 2001) with $k=10$ and performed adaptive lasso. For the complete-case analysis, because very few patients have complete data, we removed only the patients with no protein expression data; we imputed the missing values for the remaining patients using 10-nearest-neighbor imputation and performed adaptive lasso on the imputed data set. In constructing the risk scores, we again imputed missing values of $\boldsymbol{S}$ using 10-nearest-neighbor imputation.

For the complete-case analysis, the likelihood-ratio $p$-values for the association between the risk score and progression-free survival time under the stratified Cox model are 0.024 and $3.98 \times 10^{-6}$ for HNSC and LUAD, respectively. While the $p$-value for LUAD is as significant as that of the proposed method, the $p$-value for HNSC is only mildly significant. For single imputation, the likelihood-ratio $p$-values are $1.84 \times 10^{-3}$ and $1.80 \times 10^{-5}$ for HNSC and LUAD, respectively. Single imputation yields similar results as the proposed method, with slightly less significant $p$-values for both cancer types.

As a by-product of the proposed method, we obtained a low-dimensional projection of the genomic features, $\widehat{\boldsymbol{U}}$. We constructed an alternative risk score, defined as $\boldsymbol{\beta}^{\mathrm{T}} \boldsymbol{\Psi} \widehat{\boldsymbol{U}}$, which is the estimated effect of the projected genomic features on tumor stage. For each cancer type and tumor stage group, we divided patients into two equal-sized risk groups 
according to their risk scores. The Kaplan-Meier curves of the progression-free survival times for the risk groups are given in Figure 1. We also tested the association between the risk score and progression-free survival time under the stratified Cox model, and the likelihood-ratio $p$-values are $1.55 \times 10^{-5}$ and $2.22 \times 10^{-3}$ for HNSC and LUAD, respectively. Remarkably, patients classified into high-risk groups tend to have lower (progressionfree) survival probabilities than patients in the corresponding low-risk groups. Also, the likelihood-ratio tests are significant for both cancer types, and the $p$-value for HNSC is smaller than those obtained from the original risk score (using the proposed method, single imputation, or complete-case analysis). A possible explanation is that the projection of the genomic features contains less noise than the individual genomic features and thus better represents patients' genomic characteristics.
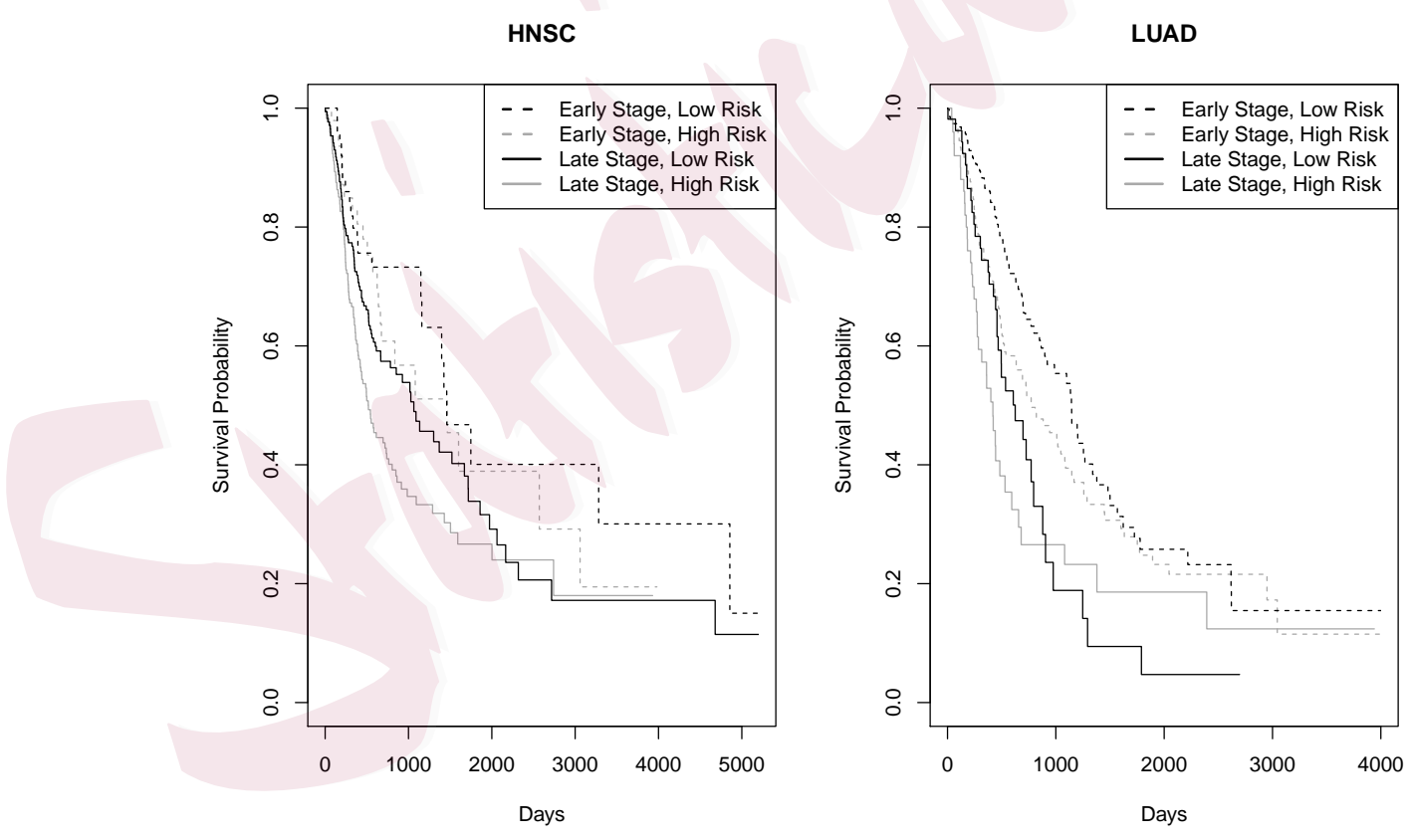

Figure 1: Kaplan-Meier curves for risk groups defined by the projected genomic features. 
Finally, we compared the three methods for handling missing data using cross validation. We first independently sampled 10 training sets from the full data set. Each training set consists of $60 \%$ of the patients $(n=573)$; the training set and the full data set have approximately equal distributions of cancer type, tumor stage, and missing-data proportion. In each training set, we followed the procedures used in the analyses of the whole data set: we screened the gene expressions by selecting the 500 gene expressions with the strongest association with tumor stage (after adjusting for cancer type) and performed complete-case analysis, single imputation by 10-nearest-neighbor imputation, and the proposed method on the screened variables over a sequence of tuning parameter values. For the proposed method, we set the numbers of latent variables in the factor model to be $r_{0}=2, r_{1}=3$, and $r_{2}=1$.

To evaluate the performance of each method, we used 10-nearest-neighbor imputation to impute the missing values in the full data set and let each validation set consist of patients that were not part of a corresponding training set. For each training and validation data split and each method, we performed the analysis using the training set over a series of tuning parameter values and computed the area under the receiver operating characteristic curve (AUC) of the resulting risk scores in the validation set. Each method produces a curve of AUC against tuning parameter values for each data split. Because the variables in the regression analyses are different in different data splits due to screening, it is not appropriate to combine the results over splits at the same tuning parameter values. Instead, we averaged the AUC values over splits at models with the same number of selected variables.

The complete-case analysis yields the smallest average AUC at any given model size, 
and the average AUC values between single imputation and the proposed method are similar. Specifically, the maximum average AUC values for the complete-case analysis, single imputation, and the proposed method are $0.802,0.816$, and 0.817 , respectively. Possibly due to the small sample size of the training data sets, the performance of single imputation and the proposed method is similar.

\section{Discussion}

In this article, we propose a penalized-likelihood approach to variable selection and parameter estimation for multiple types of many features with missing data. Our approach accommodates arbitrary missing-data patterns, including but not restricted to blockwise missing data. We prove estimation and model selection consistency of the penalized estimator and develop an efficient EM algorithm for its computation. A key advantage of the proposed estimator is that it is consistent under MAR, whereas single imputation is generally biased when the missing-data mechanism depends on the outcome variable.

The structure of the latent factor model facilitates efficient computation of the penalized estimator under general missing-data patterns. Under the factor model, the conditional distribution of $\boldsymbol{S}^{(M)}$ given $\left(\boldsymbol{U}, \boldsymbol{S}^{(O)}\right)$ is equal to the conditional distribution of $\boldsymbol{S}^{(M)}$ given $\boldsymbol{U}$ alone. This structure makes it simpler to evaluate the conditional distribution of the variables with missing values given the observed variables in the E-step. (By contrast, if an unstructured covariance matrix for $\boldsymbol{S}$ is assumed, then the conditional expectation may involve inversion of large matrices.) In addition, due to the conditional independence of components of $\boldsymbol{S}$, the factor loadings for each component of $\boldsymbol{S}$ can be updated separately with closed-form solutions in the M-step. 


\section{DISCUSSION}

Under the normality assumption on the error term $\boldsymbol{\epsilon}$ in the factor model, the EM algorithm only involves a low-dimensional numerical integration even when the dimension of the incomplete variables is high. This assumption ensures the existence of a linear transformation of $\boldsymbol{\epsilon}$, denoted by $\widetilde{\boldsymbol{\epsilon}}$, such that components of $\widetilde{\boldsymbol{\epsilon}}$ are independent, and the outcome variable depends on $\boldsymbol{\epsilon}$ only through a single component of $\widetilde{\boldsymbol{\epsilon}}$. For general distributions of $\boldsymbol{\epsilon}$, such a linear transformation is not available, and the integration in the likelihood function cannot be simplified.

We use BIC to select the numbers of common and type-specific latent variables. When the number of feature types is large, this approach may involve evaluation of a large number of models and may be computationally intensive. Alternatively, we can consider penalization approaches similar to Ibrahim et al. (2011) and Caner and Han (2014), which penalize variances of the latent variables or columns of the factor loading matrix.

One application of the proposed approach lies in dimension reduction of multi-modality features. In genomics studies, low-dimensional projections of genomic features have been used to pick out technical errors (Leek et al., 2010), characterize the activities of gene sets (Fan et al., 2011), and discover molecular subtypes of patients (Shen, Olshen and Ladanyi, 2009; Shen, Wang and Mo, 2013). Most projection methods do not account for missing data, much less a missing-not-at-random mechanism. Under the proposed framework, if the missing-data mechanism depends on an external variable (that is associated with $\boldsymbol{S}$ ), then we can set the variable as the outcome variable $Y$, and the resulting factor model can be estimated without bias and be used to generate the projection.

Our work can be extended in several directions. First, we may consider a potentially right-censored outcome variable that follows the Cox proportional hazards model. This 


\section{DISCUSSION}

extension would find applications in many multi-platform genomics studies in which the outcome of interest is time to death or disease progression. For this extension, the penalized estimator can be computed using the EM algorithm, where the M-step involves maximizing a quadratic approximation of the (expected) log-partial likelihood using the coordinate-descent algorithm (Simon et al., 2011).

Another extension is to allow associations among features beyond those explained by the latent variables. The extra associations can be accommodated by adopting an approximate factor model of $\boldsymbol{S}$, which allows nonzero but sparse off-diagonal elements in $\Sigma$. A sparse estimator of $\Sigma$ can be obtained by including a penalty term on the off-diagonal elements of the covariance matrix in the penalized likelihood (Bai and Liao, 2016). This generalization, however, imposes considerable computational challenges by complicating the conditional distribution of $\boldsymbol{S}$ given $\boldsymbol{U}$ and introducing an extra tuning parameter.

A third extension is to consider nonnormal incomplete features. We may fit a semiparametric factor model, such that each latent variable is a nonparametric monotone transformation of a Gaussian variable. We can adopt penalized sieve maximum likelihood methods for estimation and the EM algorithm for computation. Another possibility is to assume that transformations of components of $\boldsymbol{S}$ follow the (Gaussian) factor model and fit a regression model of $Y$ on the transformed $\boldsymbol{S}$.

Finally, we have only established estimation and selection consistency of the penalized estimator. It is plausible that the estimator for the nonzero parameters exhibits the so-called oracle properties (Fan and Li, 2001) under further regularity conditions. However, inference based on the oracle properties ignores the variability arising from variable 
selection and thus is seldom conducted in practice. A possible future research direction is to investigate a "de-biased" version of the penalized estimator van de Geer et al. 2014; Zhang and Zhang, 2014), which yields more reliable inference at the expense of nonsparsity of the estimators.

\section{Supplementary Materials}

The supplementary materials contain additional theoretical results, simulation results, and details of the real data analysis.

\section{Acknowledgements}

This research was supported by a start-up research grant from the Hong Kong Polytechnic University (1-BE02), the Hong Kong Research Grants Council grant PolyU 253042/18P, and the National Institutes of Health grants R01HG009974, R01GM047845, and P01CA142538.

\section{Appendix A: Computational and technical details}

\section{A.1 Details of the EM algorithm}

To express the first and second conditional moments of $\widetilde{\boldsymbol{S}}_{i}$ given $\mathcal{O}_{i} \equiv\left(Y_{i}, \boldsymbol{X}_{i}, \boldsymbol{S}_{i}^{(O)}\right)$ in terms of those of $\left(\boldsymbol{U}_{i}, \widetilde{\epsilon}_{i}\right)$, we define $\widetilde{\boldsymbol{\beta}}_{i}^{(M)}=\left(\boldsymbol{\beta}_{i}^{(M)}\right)_{j: \beta_{i j}^{(M)} \neq 0}, \widetilde{\boldsymbol{\sigma}}_{i}^{(M)}=\left(\boldsymbol{\sigma}_{i}^{(M)}\right)_{j: \beta_{i j}^{(M)} \neq 0}$, and $\widetilde{p}_{i}^{(M)}$ to be the dimension of $\widetilde{\boldsymbol{\beta}}_{i}^{(M)}$, where $\boldsymbol{\sigma}_{i}^{(M)}=\left(\sigma_{1}, \ldots, \sigma_{p_{n}}\right)_{\mathcal{M}_{i}}^{\mathrm{T}}$. Let $\widetilde{\boldsymbol{\vartheta}}_{i 1}=$ $c_{i}^{-1}\left(\widetilde{\beta}_{i 1}^{(M)} \widetilde{\sigma}_{i 1}^{(M)}, \ldots, \widetilde{\beta}_{i \widetilde{p}^{(M)}}^{(M)} \widetilde{\sigma}_{i \widetilde{p}^{(M)}}^{(M)}\right)^{\mathrm{T}}$, and set $\left(\widetilde{\boldsymbol{\vartheta}}_{i 2}, \ldots, \widetilde{\boldsymbol{\vartheta}}_{i \widetilde{p}_{i}^{(M)}}\right)$ to be $\widetilde{p}_{i}^{(M)}$-dimensional unit vectors that are orthogonal to $\widetilde{\boldsymbol{\vartheta}}_{i 1}$ and to each other. The first and second (conditional) moments of $\widetilde{\boldsymbol{S}}_{i}$ involve $\mathrm{E}\left(\boldsymbol{U}_{i} \mid \mathcal{O}_{i}\right), \mathrm{E}\left(\boldsymbol{\epsilon}_{i}^{(M)} \mid \mathcal{O}_{i}\right), \mathrm{E}\left(\boldsymbol{U}_{i} \boldsymbol{U}_{i}^{\mathrm{T}} \mid \mathcal{O}_{i}\right), \mathrm{E}\left(\widehat{\boldsymbol{\Psi}}_{i}^{(M)} \boldsymbol{U}_{i} \boldsymbol{\epsilon}_{i}^{(M) \mathrm{T}} \mid \mathcal{O}_{i}\right)$, 
and $\mathrm{E}\left(\boldsymbol{\epsilon}_{i}^{(M)} \boldsymbol{\epsilon}_{i}^{(M) \mathrm{T}} \mid \mathcal{O}_{i}\right)$. The moments of $\boldsymbol{U}_{i}$ are readily available from 3.4 by setting $g\left(\boldsymbol{U}_{i}, \widetilde{\epsilon}_{i}\right)=\boldsymbol{U}_{i}$ or $\boldsymbol{U}_{i} \boldsymbol{U}_{i}^{\mathrm{T}}$. For the other terms in the moments of $\widetilde{\boldsymbol{S}}_{i}$, we have

$$
\begin{aligned}
& \mathrm{E}\left(\epsilon_{i j} \mid \mathcal{O}_{i}\right)= \begin{cases}c_{i}^{-1} \sigma_{i j}^{(M)} \widetilde{\vartheta}_{i 1, m_{i}(j)} \mathrm{E}\left(\widetilde{\epsilon}_{i} \mid \mathcal{O}_{i}\right) & \text { if } \beta_{i j}^{(M)} \neq 0 \\
0 & \text { otherwise }\end{cases} \\
& \mathrm{E}\left(\widehat{\boldsymbol{\Psi}}_{i}^{(M)} \boldsymbol{U}_{i} \boldsymbol{\epsilon}_{i}^{(M) \mathrm{T}} \mid \mathcal{O}_{i}\right)_{j k}= \begin{cases}c_{i}^{-1} \sigma_{i k}^{(M)} \widetilde{\vartheta}_{i 1, m_{i}(k)} \sum_{h=1}^{r} \widehat{\psi}_{i j h}^{(M)} \mathrm{E}\left(U_{i h} \widetilde{\epsilon}_{i} \mid \mathcal{O}_{i}\right) & \text { if } \beta_{i k}^{(M)} \neq 0 \\
0 & \text { otherwise }\end{cases} \\
& \mathrm{E}\left(\boldsymbol{\epsilon}_{i}^{(M)} \boldsymbol{\epsilon}_{i}^{(M) \mathrm{T}} \mid \mathcal{O}_{i}\right)_{j k}= \begin{cases}c_{i}^{-2} \sigma_{i j}^{(M)} \sigma_{i k}^{(M)} \delta_{j k} & \text { if } \beta_{i j}^{(M)}=0 \text { or } \beta_{i k}^{(M)}=0, \\
c_{i}^{-2} \sum_{h=1}^{\widetilde{p}_{i}^{(M)}}\left\{I(h=1) \mathrm{E}\left(\widetilde{\epsilon}_{i}^{2} \mid \mathcal{O}_{i}\right)\right. & \\
+I(h \neq 1)\} \sigma_{i j}^{(M)} \sigma_{i k}^{(M)} \widetilde{\vartheta}_{i h, m_{i}(j)} \widetilde{\vartheta}_{i h, m_{i}(k)} & \text { otherwise, }\end{cases}
\end{aligned}
$$

where $\widetilde{\vartheta}_{i j k}$ is the $k$ th component of $\widetilde{\boldsymbol{\vartheta}}_{i j}, m_{i}(j)$ is such that $\widetilde{\beta}_{i, m_{i}(j)}^{(M)}=\beta_{i j}^{(M)}, \widehat{\psi}_{i j k}^{(M)}$ is the $(j, k)$ th element of $\widehat{\Psi}_{i}^{(M)}$, and $\delta_{j k}=I(j=k)$.

\section{A.2 Proofs of technical results}

Before proving Theorem 1, we present the following lemma, which pertains to an estimator of the nonzero parameters. Let $\widehat{\boldsymbol{\theta}}_{\text {Oracle }} \equiv\left(\widehat{\boldsymbol{\alpha}}_{\text {Oracle }}, \widehat{\boldsymbol{\beta}}_{\text {Oracle }}, \widehat{\boldsymbol{\xi}}_{\text {Oracle }}, \widehat{\boldsymbol{\Gamma}}_{\text {Oracle }}, \widehat{\boldsymbol{\Psi}}_{\text {Oracle }}, \widehat{\boldsymbol{\Sigma}}_{\text {Oracle }}\right)$ be a local maximizer of $p \ell_{n}(\boldsymbol{\theta})$ when $\boldsymbol{\beta}_{\mathcal{N}}$ is fixed at $\mathbf{0}$. For any potentially random realvalued sequences $a_{n}$ and $b_{n}$, we say that $a_{n}$ is dominated by $b_{n}$ if $\left|a_{n} / b_{n}\right|=o_{p}(1)$. We have the following result about the oracle estimator.

Lemma 1. Under conditions $(C 1)-(C 6)$, there is a version of $\widehat{\boldsymbol{\theta}}_{\text {Oracle }}$ that satisfies

$$
\left\|\widehat{\boldsymbol{\alpha}}_{\text {Oracle }}-\boldsymbol{\alpha}_{0}\right\|+\left\|\widehat{\boldsymbol{\beta}}_{\text {Oracle }}-\boldsymbol{\beta}_{0}\right\|+\left\|\widehat{\boldsymbol{\xi}}_{\text {Oracle }}-\boldsymbol{\xi}_{0}\right\|+p_{n}^{-1 / 2}\left\|\boldsymbol{H} \boldsymbol{\Sigma}_{0}^{-1 / 2}\left(\widehat{\boldsymbol{\Gamma}}_{\text {Oracle }}-\boldsymbol{\Gamma}_{0}\right)\right\|
$$




$$
\begin{aligned}
& +\left\|(\boldsymbol{I}-\boldsymbol{H}) \boldsymbol{\Sigma}_{0}^{-1 / 2}\left(\widehat{\boldsymbol{\Gamma}}_{\text {Oracle }}-\boldsymbol{\Gamma}_{0}\right)\right\|+p_{n}^{-1 / 2}\left\|\boldsymbol{H} \boldsymbol{\Sigma}_{0}^{-1 / 2}\left(\widehat{\Psi}_{\text {Oracle }}-\boldsymbol{\Psi}_{0}\right)\right\| \\
& +\left\|(\boldsymbol{I}-\boldsymbol{H}) \boldsymbol{\Sigma}_{0}^{-1 / 2}\left(\widehat{\boldsymbol{\Psi}}_{\text {Oracle }}-\boldsymbol{\Psi}_{0}\right)\right\|+\left\|\widehat{\boldsymbol{\Sigma}}_{\text {Oracle }}-\boldsymbol{\Sigma}_{0}\right\|=O_{p}\left(n^{-1 / 2} p_{n}^{1 / 2}\right) .
\end{aligned}
$$

The proof of Lemma 1 is presented in Section S3 of the supplementary materials.

Proof of Theorem 1. By Lemma 1, $\widehat{\boldsymbol{\theta}}_{\text {Oracle }}$ converges in probability to $\boldsymbol{\theta}_{0}$ at the desired rate of convergence, so it suffices to show that there exists a local maximizer of $p \ell_{n}(\boldsymbol{\theta})$, denoted by $\widehat{\boldsymbol{\theta}}$, such that $P\left(\widehat{\boldsymbol{\theta}}=\widehat{\boldsymbol{\theta}}_{\text {Oracle }}\right) \rightarrow 1$ for some version of the oracle estimator

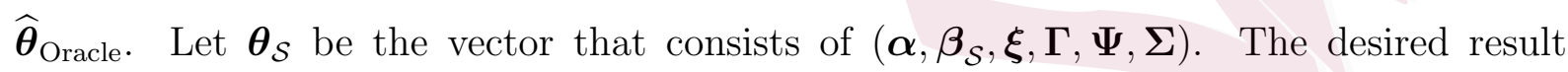
follows if the following Karush-Kuhn-Tucker conditions hold:

$$
\begin{aligned}
&\left.\left\{\mathbb{P}_{n} \frac{\partial}{\partial \boldsymbol{\theta}_{\mathcal{S}}} \ell(\boldsymbol{\theta})-\lambda_{n} \boldsymbol{w}_{\mathcal{S}} \circ \operatorname{sgn}\left(\boldsymbol{\beta}_{\mathcal{S}}\right)\right\}\right|_{\boldsymbol{\theta}=\widehat{\boldsymbol{\theta}}_{\text {Oracle }}}=\mathbf{0}, \\
&\left|\mathbb{P}_{n} \frac{\partial}{\partial \beta_{j}} \ell(\boldsymbol{\theta})\right|_{\boldsymbol{\theta}=\widehat{\boldsymbol{\theta}}_{\text {Oracle }} \mid} \mid<\lambda_{n} w_{j} \quad \text { for } j=p_{1 n}+1, \ldots, p_{n}, \\
& \lambda_{\min }\left\{-\left.\mathbb{P}_{n} \frac{\partial^{2}}{\partial \boldsymbol{\theta}_{\mathcal{S}} \partial \boldsymbol{\theta}_{\mathcal{S}}^{\mathrm{T}}} \ell(\boldsymbol{\theta})\right|_{\boldsymbol{\theta}=\widehat{\boldsymbol{\theta}}_{\text {Oracle }}}\right\}>0,
\end{aligned}
$$

where $\ell(\boldsymbol{\theta})$ denotes the log-likelihood function for a single subject, $\mathbb{P}_{n}$ denotes the empirical process measure, and $\operatorname{sgn}\left(\boldsymbol{\beta}_{\mathcal{S}}\right)$ is the vector of the signs of $\boldsymbol{\beta}_{\mathcal{S}}$. The first condition holds by the definition of $\widehat{\boldsymbol{\theta}}_{\text {Oracle }}$, and the third condition follows from condition (C1) and the fact that the Hessian matrix of the $\log$-likelihood function with respect to $(\boldsymbol{\Gamma}, \boldsymbol{\Psi}, \boldsymbol{\Sigma})$ is negative definite for large enough $n$ (as established in the proof of Lemma 1). To verify the second condition, let $u_{n}=n^{\tau+\eta+\kappa-1}$ for some $\eta<1-\kappa$, and let

$$
\mathcal{S}_{n}=\left\{\left|n^{1 / 2} \frac{\partial}{\partial \beta_{j}} \mathbb{P}_{n} \ell\left(\boldsymbol{\theta}_{0}\right)\right| \leq u_{n} \quad \text { for } j=p_{1 n}+1, \ldots, p_{n}\right\} .
$$


For $k \geq 1$ and $j=p_{1 n}+1, \ldots, p_{n}$,

$$
\begin{aligned}
\left.\mathrm{E}\left|\frac{\partial}{\partial \beta_{j}} \ell(\boldsymbol{\theta})\right|_{\boldsymbol{\theta}=\boldsymbol{\theta}_{0}}\right|^{k} & =\mathrm{E}\left|\int Z\left(\boldsymbol{\alpha}_{0}^{\mathrm{T}} \boldsymbol{X}+\boldsymbol{\beta}_{0}^{\mathrm{T}} \boldsymbol{S}, \boldsymbol{\xi}_{0}\right) S_{j} f\left(\boldsymbol{S}^{(M)} \mid \boldsymbol{S}^{(O)}, Y, \boldsymbol{X}\right) \mathrm{d} \boldsymbol{S}^{(M)}\right|^{k} \\
& \leq \mathrm{E}\left|Z\left(\boldsymbol{\alpha}_{0}^{\mathrm{T}} \boldsymbol{X}+\boldsymbol{\beta}_{0}^{\mathrm{T}} \boldsymbol{S}, \boldsymbol{\xi}_{0}\right) S_{j}\right|^{k}
\end{aligned}
$$

where $f\left(\boldsymbol{S}^{(M)} \mid \boldsymbol{S}^{(O)}, Y, \boldsymbol{X}\right)$ is the true conditional density function of $\boldsymbol{S}^{(M)}$ given $\left(\boldsymbol{S}^{(O)}, Y, \boldsymbol{X}\right)$, and the inequality follows from Jensen's inequality. By condition (C7) and the Bernstein inequality, there exists some fixed positive constants $c_{0}$ and $c_{1}$ such that

$$
P\left(\left|n^{1 / 2} \frac{\partial}{\partial \beta_{j}} \mathbb{P}_{n} \ell(\boldsymbol{\theta})\right|_{\boldsymbol{\theta}=\boldsymbol{\theta}_{0}} \mid>u_{n}\right) \leq c_{0} e^{-c_{1} u_{n}}
$$

for $j=p_{1 n}+1, \ldots, p_{n}$. Therefore, $P\left(\mathcal{S}_{n}\right) \geq 1-c_{0} p_{n} e^{-c_{1} u_{n}}=1-O\left(e^{\kappa \log n-c_{1} u_{n}}\right)$, which tends to 1 because, by conditions (C3) and (C8), $\eta$ can be chosen such that $u_{n}$ dominates $\log n$. Under $\mathcal{S}_{n}$ and for $\widetilde{\boldsymbol{\theta}}$ such that the $\boldsymbol{\theta}_{\mathcal{S}^{-}}$component of $\widetilde{\boldsymbol{\theta}}$ is close enough to its true value and the $\boldsymbol{\beta}_{\mathcal{N}^{-}}$component of $\widetilde{\boldsymbol{\theta}}$ is zero,

$$
\begin{aligned}
\left.\frac{\partial}{\partial \beta_{j}} \mathbb{P}_{n} \ell(\boldsymbol{\theta})\right|_{\boldsymbol{\theta}=\widetilde{\boldsymbol{\theta}}} & =\left.\frac{\partial}{\partial \beta_{j}} \mathbb{P}_{n} \ell(\boldsymbol{\theta})\right|_{\boldsymbol{\theta}=\boldsymbol{\theta}_{0}}+\left.\frac{\partial^{2}}{\partial \beta_{j} \partial \boldsymbol{\theta}_{\mathcal{S}}^{\mathrm{T}}} \mathbb{P}_{n} \ell(\boldsymbol{\theta})\right|_{\boldsymbol{\theta}=\check{\boldsymbol{\theta}}}\left(\widetilde{\boldsymbol{\theta}}-\boldsymbol{\theta}_{0}\right) \\
& \leq n^{-1 / 2} u_{n}+\sup _{\boldsymbol{\theta}^{*}}\left\|\left.\frac{\partial^{2}}{\partial \beta_{j} \partial \boldsymbol{\theta}_{\mathcal{S}}^{\mathrm{T}}} \mathbb{P}_{n} \ell(\boldsymbol{\theta})\right|_{\boldsymbol{\theta}=\boldsymbol{\theta}^{*}}\left(\widetilde{\boldsymbol{\theta}}-\boldsymbol{\theta}_{0}\right)\right\| \\
& \leq n^{-1 / 2} u_{n}+\sup _{\boldsymbol{\theta}^{*}}\left\|\left.\frac{\partial^{2}}{\partial \boldsymbol{\beta}_{\mathcal{N}} \partial \boldsymbol{\theta}_{\mathcal{S}}^{\mathrm{T}}} \mathbb{P}_{n} \ell(\boldsymbol{\theta})\right|_{\boldsymbol{\theta}=\boldsymbol{\theta}^{*}}\right\|\left\|_{2, \infty}\right\| \widetilde{\boldsymbol{\theta}}-\boldsymbol{\theta}_{0} \|
\end{aligned}
$$

for $j=p_{1 n}+1, \ldots, p_{n}$ and large enough $n$, where $\check{\boldsymbol{\theta}}$ takes some value between $\widetilde{\boldsymbol{\theta}}$ and $\boldsymbol{\theta}_{0}$, and the supremum is taken within the small neighborhood around $\boldsymbol{\theta}_{0}$ defined in condition (C8). Note that

$$
\begin{aligned}
\sup _{\boldsymbol{\theta}^{*}}\left\|\left.\frac{\partial^{2}}{\partial \boldsymbol{\beta}_{\mathcal{N}} \partial \boldsymbol{\theta}_{\mathcal{S}}^{\mathrm{T}}} \mathbb{P}_{n} \ell(\boldsymbol{\theta})\right|_{\boldsymbol{\theta}=\boldsymbol{\theta}^{*}}\right\|_{2, \infty} & =\sup _{\boldsymbol{\theta}^{*}}\left\|\left.\frac{\partial^{2}}{\partial \boldsymbol{\beta}_{\mathcal{N}} \partial \boldsymbol{\theta}_{\mathcal{S}}^{\mathrm{T}}} \mathbb{P} \ell(\boldsymbol{\theta})\right|_{\boldsymbol{\theta}=\boldsymbol{\theta}^{*}}\right\|_{2, \infty}+O_{p}\left(n^{-1 / 2} p_{n}^{2}\right) \\
& =\sup _{\boldsymbol{\theta}^{*}}\left\|\boldsymbol{I}\left(\boldsymbol{\theta}^{*}\right)\right\|_{2, \infty}+o_{p}(1) \\
& =O_{p}\left(n^{\tau+\eta-1}\right)
\end{aligned}
$$


where the third equality follows from condition (C8), and $\mathbb{P}$ is the true probability measure. Therefore,

$$
\begin{aligned}
\max _{j>p_{1 n}}\left|\lambda_{n}^{-1} w_{j}^{-1} \frac{\partial}{\partial \beta_{j}} \mathbb{P}_{n} \ell(\boldsymbol{\theta})\right|_{\boldsymbol{\theta}=\widehat{\boldsymbol{\theta}}_{\text {Oracle }} \mid} \mid & \leq \max _{j>p_{1 n}} O_{p}\left(\lambda_{n}^{-1} w_{j}^{-1} n^{-1 / 2} u_{n}+\lambda_{n}^{-1} w_{j}^{-1} n^{\tau+\eta-1} p_{n} n^{-1 / 2}\right) \\
& =O_{p}\left(\lambda_{n}^{-1} n^{-\tau} n^{-1 / 2} u_{n}+\lambda_{n}^{-1} n^{\eta-3 / 2} n^{\kappa}\right),
\end{aligned}
$$

where the equality follows from condition (C3). The right-hand side of the equality above is bounded by $O_{p}\left(\lambda_{n}^{-1} n^{-3 / 2+\kappa+\eta}\right)$, which is $o_{p}(1)$ by condition (C9). The desired result

follows.

\section{References}

Bai, J. (2003). Inferential theory for factor models of large dimensions. Econometrica 71, 135-171.

Bai, J. and Li, K. (2012). Statistical analysis of factor models of high dimension. Ann. Statist. 40, 436-465.

Bai, J. and Liao, Y. (2016). Efficient estimation of approximate factor models via penalized maximum likelihood.

\section{J. Econometrics 191, 1-18.}

Cai, T., Cai, T. T. and Zhang, A. (2016). Structured matrix completion with applications to genomic data integration. J. Amer. Statist. Assoc. 111, 621-633.

Caner, M. and Han, X. (2014). Selecting the correct number of factors in approximate factor models: the large panel case with group bridge estimators. J. Bus. Econom. Statist. 32, 359-374.

Dempster, A. P., Laird, N. M. and Rubin, D. B. (1977). Maximum likelihood from incomplete data via the EM algorithm. J. R. Stat. Soc. Ser. B. Stat. Methodol. 39, 1-22.

Fan, C., Prat, A., Parker, J. S., Liu, Y., Carey, L. A., Troester, M. A. and Perou, C. M. (2011). Building prognostic models for breast cancer patients using clinical variables and hundreds of gene expression signatures. $B M C$ Medical Genom. 4, 3. 
Fan, J. and Li, R. (2001). Variable selection via nonconcave penalized likelihood and its oracle properties. J. Amer. Statist. Assoc. 96, 1348-1360.

Fan, J., Liao, Y. and Mincheva, M. (2013). Large covariance estimation by thresholding principal orthogonal complements. J. R. Stat. Soc. Ser. B. Stat. Methodol. 75, 603-680.

Fan, J., Liu, H. and Wang, W. (2018). Large covariance estimation through elliptical factor models. Ann. Statist. 46, 1383-1414.

Fan, J. and Lv, J. (2011). Nonconcave penalized likelihood with NP-dimensionality. IEEE Trans. Inform. Theory 57, 5467-5484.

Fan, J. and Peng, H. (2004). Nonconcave penalized likelihood with a diverging number of parameters. Ann. Statist. 32, 928-961.

Fan, J. and Song, R. (2010). Sure independence screening in generalized linear models with NP-dimensionality. Ann. Statist. 38, 3567-3604.

Friedman, J., Hastie, T. and Tibshirani, R. (2010). Regularization paths for generalized linear models via coordinate descent. J. Stat. Softw. 33, 1-22.

Garcia, R. I., Ibrahim, J. G. and Zhu, H. (2010). Variable selection for regression models with missing data. Statist. Sinica 20, 149-165.

Hanahan, D. and Weinberg, R. A. (2011). Hallmarks of cancer: the next generation. Cell 144, 646-674.

Heiss, F. and Winschel, V. (2008). Likelihood approximation by numerical integration on sparse grids. $J$. Econometrics 144, 62-80.

Hoadley, K. A., Yau, C., Wolf, D. M., Cherniack, A. D., Tamborero, D., Ng, S., Leiserson, M. D. M., Niu, B., McLellan, M. D., Uzunangelov, V., Zhang, J., Kandoth, C., Akbani, R., Shen, H., Omberg, L., Chu, A., Margolin, A. A., van't Veer, L. J., Lopez-Bigas, N., Laird, P. W., Raphael, B. J., Ding, L., Robertson, A. G., 
Byers, L. A., Mills, G. B., Weinstein, J. N., Van Waes, C., Chen, Z., Collisson, E. A., The Cancer Genome Atlas Research Network, Benz, C. C., Perou, C. M. and Stuart, J. M. (2014). Multiplatform analysis of 12 cancer types reveals molecular classification within and across tissues of origin. Cell 158, 929-944.

Ibrahim, J. G., Zhu, H., Garcia, R. I. and Guo, R. (2011). Fixed and random effects selection in mixed effects models. Biometrics 67, 495-503.

Ibrahim, J. G., Zhu, H. and Tang, N. (2008). Model selection criteria for missing-data problems using the EM algorithm. J. Amer. Statist. Assoc. 103, 1648-1658.

Jiang, J., Nguyen, T. and Rao, J. S. (2015). The E-MS algorithm: model selection with incomplete data. J. Amer. Statist. Assoc. 110, 1136-1147.

Läubli, H., Stevenson, J. L., Varki, A., Varki, N. M. and Borsig, L. (2006). L-selectin facilitation of metastasis involves temporal induction of Fut7-dependent ligands at sites of tumor cell arrest. Cancer Res. 66, 15361542 .

Leek, J. T., Scharpf, R. B., Bravo, H. C., Simcha, D., Langmead, B., Johnson, W. E., Geman, D., Baggerly, K. and Irizarry, R. A. (2010). Tackling the widespread and critical impact of batch effects in high-throughput data. Nat. Rev. Genet. 11, 733-739.

Liu, Q. and Pierce, D. A. (1994). A note on Gauss-Hermite quadrature. Biometrika 81, 624-629.

Lock, E. F., Hoadley, K. A., Marron, J. S. and Nobel, A. B. (2013). Joint and individual variation explained (JIVE) for integrated analysis of multiple data types. Ann. Appl. Stat. 7, 523-542.

Nicholson, R. I., Gee, J. M. W. and Harper, M. E. (2001). EGFR and cancer prognosis. Eur. J. Cancer 37, S9-S15.

Normanno, N., De Luca, A., Bianco, C., Strizzi, L., Mancino, M., Maiello, M. R., Carotenuto, A., De Feo, G., Caponigro, F. and Salomon, D. S. (2006). Epidermal growth factor receptor (EGFR) signaling in cancer. 
Gene 366, 2-16.

Ogawa, J., Inoue, H. and Koide, S. (1997). $\alpha$-2,3-sialyltransferase type $3 \mathrm{~N}$ and $\alpha$-1,3-fucosyltransferase type VII are related to sialyl Lewis ${ }^{x}$ synthesis and patient survival from lung carcinoma. Cancer 79, 1678-1685.

Schwarz, G. (1978). Estimating the dimension of a model. Ann. Statist. 6, 461-464.

Shen, R., Olshen, A. B. and Ladanyi, M. (2009). Integrative clustering of multiple genomic data types using a joint latent variable model with application to breast and lung cancer subtype analysis. Bioinformatics $\mathbf{2 5}$, $2906-2912$.

Shen, R., Wang, S. and Mo, Q. (2013). Sparse integrative clustering of multiple omics data sets. Ann. Appl. Stat. 7, 269-294.

Simon, N., Friedman, J., Hastie, T. and Tibshirani, R. (2011). Regularization paths for Cox's proportional hazards model via coordinate descent. J. Stat. Softw. 39, 1-13.

Tibshirani, R. (1996). Regression shrinkage and selection via the lasso. J. R. Stat. Soc. Ser. B. Stat. Methodol. 58, $267-288$.

Troyanskaya, O., Cantor, M., Sherlock, G., Brown, P., Hastie, T., Tibshirani, R., Botstein, D. and Altman, R. B. (2001). Missing value estimation methods for DNA microarrays. Bioinformatics 17, 520-525.

van de Geer, S., Bühlmann, P., Ritov, Y. and Dezeure, R. (2014). On asymptotically optimal confidence regions and tests for high-dimensional models. Ann. Statist. 42, 1166-1202.

Wang, W., Baladandayuthapani, V., Morris, J. S., Broom, B. M., Manyam, G. and Do, K.-A. (2012). iBAG: integrative Bayesian analysis of high-dimensional multiplatform genomics data. Bioinformatics 29, 149-159.

Wang, Y., Han, E., Xing, Q., Yan, J., Arrington, A., Wang, C., Tully, D., Kowolik, C. M., Lu, D. M., Frankel, P. H., Zhai, J., Wen, W., Horne, D., Yip, M. L. R. and Yim, J. H. (2015). Baicalein upregulates DDIT4 expression which mediates mTOR inhibition and growth inhibition in cancer cells. Cancer Lett. 358, 170-179. 
Wong, K. Y., Fan, C., Tanioka, M., Parker, J. S., Nobel, A. B., Zeng, D., Lin, D. Y. and Perou, C. M. (2019). I-Boost: an integrative boosting approach for predicting survival time with multiple genomics platforms. Genome Biol. 20, 52 .

Zhang, C.-H. and Zhang, S. S. (2014). Confidence intervals for low dimensional parameters in high dimensional linear models. J. R. Stat. Soc. Ser. B. Stat. Methodol. 76, 217-242.

Zhang, D., Shen, D. and The Alzheimer's Disease Neuroimaging Initiative (2012). Multi-modal multi-task learning for joint prediction of multiple regression and classification variables in Alzheimer's disease. NeuroImage 59, $895-907$.

Zhao, P. and Yu, B. (2006). On model selection consistency of Lasso. J. Mach. Learn. Res. 7, 2541-2563.

Zou, H. (2006). The adaptive lasso and its oracle properties. J. Amer. Statist. Assoc. 101, 1418-1429.

Kin Yau Wong

Department of Applied Mathematics, The Hong Kong Polytechnic University, Hung Hom, Kowloon, Hong Kong

E-mail: kin-yau.wong@polyu.edu.hk

Donglin Zeng

Department of Biostatistics, University of North Carolina, Chapel Hill, NC 27599, USA

E-mail: dzeng@email.unc.edu

D. Y. Lin

Department of Biostatistics, University of North Carolina, Chapel Hill, NC 27599, USA

E-mail: lin@bios.unc.edu 